Supporting Information for

\title{
A Structurally Perfect S = 1/2 Metal-Organic Kagomé Antiferromagnet
}

\author{
Emily A. Nytko, Joel S. Helton, Peter Müller, and Daniel G. Nocera* \\ Contribution from the Departments of Chemistry and Physics, Massachusetts Institute of \\ Technology, 77 Massachusetts Ave. Cambridge, Massachusetts 02139-4307 \\ nocera@mit.edu
}

Experimental Section (synthesis, structure determination, FTIR, TGA) S2

$\begin{array}{ll}\text { Magnetic Characterization of } \mathrm{Cu}(1,3-\mathrm{bdc}) & \text { S8 }\end{array}$

X-ray structural data for $\mathbf{1}, \mathrm{Cu}(1,3-\mathrm{bdc}) \quad \mathrm{S} 11$

$\begin{array}{ll}\text { X-ray structural data for } 2, \mathrm{Cu}(1,3-\mathrm{bdc}) \cdot 0.11 \mathrm{H}_{2} \mathrm{O} & \mathrm{S} 17\end{array}$

$\begin{array}{ll}\text { X-ray structural data for } 3, \mathrm{C}_{32} \mathrm{H}_{24} \mathrm{Cu}_{6} \mathrm{O}_{26} & \mathrm{~S} 22\end{array}$ 


\section{Experimental Section}

Sample Preparation. Water was distilled and deionized with a Milli-Q filtering system. All other reagents were obtained from commercial vendors and used without further purification: $\mathrm{Cu}(\mathrm{OH})_{2}(99.5+\%$, Noah Technologies); $\mathrm{Cu}(\mathrm{OH}) \mathrm{F}$ (99.999\%, Alfa Aesar); imidazole (99+\%, Aldrich); and isophthalic acid (99\%, Aldrich). Hydrothermal reactions were carried out under autogeneous pressure in $23 \mathrm{~mL}$ Teflon-lined pressure vessels purchased from Parr Instruments; large-scale sample preparation was carried out in a custom-built $1900 \mathrm{~mL}$ vessel. Fisher Isotemp programmable ovens with forced-air circulation were used to obtain the desired temperature profiles for the hydrothermal reactions. Elemental analysis was performed by $\mathrm{H}$. Kolbe Mikroanalytisches Laboratorium.

Cu(1,3-bdc) (1). A $1900 \mathrm{~mL}$ liner was charged with $6.04 \mathrm{~g}(61.9 \mathrm{mmol})$ of $\mathrm{Cu}(\mathrm{OH})_{2}, 2.06 \mathrm{~g}$ (20.6 mmol) of $\mathrm{Cu}(\mathrm{OH}) \mathrm{F}, 13.71 \mathrm{~g}(82.5 \mathrm{mmol})$ of isophthalic acid, $2.81 \mathrm{~g}$ (41.3 mmol) of imidazole, and $410 \mathrm{~mL}$ of water, capped and placed into a steel hydrothermal bomb. The bomb was heated to $150{ }^{\circ} \mathrm{C}$ at a rate of $1{ }^{\circ} \mathrm{C} / \mathrm{min}$, maintained at this temperature for 5 days, and cooled to room temperature at a rate of $0.1^{\circ} \mathrm{C} / \mathrm{min}$. Blue hexagonal crystals of $\mathrm{Cu}(1,3-\mathrm{bdc})(\mathbf{1})$ lined the walls of the vessel and intimately covered a blue-green powder and were difficult to separate mechanically from the fine powder. The product mixture, therefore, was sonicated in its mother liquor in a $1000 \mathrm{~mL}$ glass bottle for 80 minutes. The suspension of powder was decanted, leaving behind blue hexagonal crystals of $\mathbf{1}$, and the powder was separated from the mother liquor by vacuum filtration. The powder was identified by pXRD to consist of starting materials $\left(\mathrm{Cu}(\mathrm{OH})_{2}\right.$, spertinite, PDF \# 80-0656; $\mathrm{Cu}(\mathrm{OH}) \mathrm{F}, \mathrm{PDF} \# 07-0306$; and isophthalic acid, PDF \#45-1541) and a green impurity, $\mathrm{C}_{32} \mathrm{H}_{24} \mathrm{Cu}_{6} \mathrm{O}_{26}$ (3), whose structure is detailed below. The filtrate mother liquor was recombined with the blue hexagonal crystals, sonicated for an additional 25 minutes, and powder suspension was again decanted from the crystals. The blue hexagonal crystals of 1 (13.3 $\mathrm{g}, \sim 71 \%$ ) were then washed with deionized water, isolated by filtration, and dried in air. Impurities were further removed by physical separation under a microscope. The purity of the 
crystalline $\mathrm{Cu}(1,3-\mathrm{bdc})$ (1) sample was judged by $\mathrm{pXRD}$. The crystal structure of $\mathbf{1}$ contains a solvent-accessible void of $45 \AA^{3}$, which can be occupied by an incidental solvent molecule. Another crystal within the same batch refined as $\mathrm{Cu}(1,3-\mathrm{bdc}) \cdot 0.11 \mathrm{H}_{2} \mathrm{O}(2)$, with $1 / 9$ of a water molecule per formula unit. Further crystallographic details are provided (vide infra). Thermogravimetric analysis (TGA) of the crystalline sample (vide infra) showed loss of $0.65 \%$ by mass (or $\sim 0.08 \mathrm{H}_{2} \mathrm{O}$ per formula unit) to a temperature of $257^{\circ} \mathrm{C}$, followed by decomposition of the sample at $300{ }^{\circ} \mathrm{C}$, leaving a residue of $\mathrm{Cu}_{2} \mathrm{O}$ (PDF \# 00-0865) and $\mathrm{Cu}$ metal (PDF \# 040836) as determined by pXRD. Prior to magnetic measurements, samples were dried by heating them uncovered in a ceramic crucible at $10{ }^{\circ} \mathrm{C} / \mathrm{min}$, maintaining them at $240{ }^{\circ} \mathrm{C}$ for $2 \mathrm{~h}$, and then cooling them to room temperature at $10{ }^{\circ} \mathrm{C} / \mathrm{min}$. No significant sample decomposition occurred, as judged by pXRD and elemental analysis. Anal. Calcd. for $\mathrm{C}_{8} \mathrm{H}_{4} \mathrm{CuO}_{4}: \mathrm{C}, 42.21 ; \mathrm{H}, 1.77 ; \mathrm{Cu}$, 27.91. Found: C, 41.90; H, 1.87; Cu, 27.74.

TGA (vide infra) of sample dried in this manner showed no significant loss of mass before sample decomposition, indicating removal of the incidental solvent.

X-ray Structure Determinations. Structures were determined for $\mathrm{Cu}(1,3-\mathrm{bdc})(\mathbf{1}), \mathrm{Cu}(1,3$ bdc) $0.11 \mathrm{H}_{2} \mathrm{O}(2)$, and $\mathrm{C}_{32} \mathrm{H}_{24} \mathrm{Cu}_{6} \mathrm{O}_{26}$ (3). Single crystals were transferred onto a microscope slide coated with mineral oil. Crystals were affixed to a glass fiber or a cryoloop using the oil, frozen in a cold nitrogen gas stream, and optically centered. The data were collected on a Siemens three-circle platform goniometer equipped with a Bruker Smart Apex CCD detector with graphite-monochromated Mo K $\alpha$ radiation $(\lambda=0.71073 \AA)$, using both phi and omega scans at $-173^{\circ} \mathrm{C}$. Data reduction and unit cell refinements were performed with the program SAINT, ${ }^{1}$ and the program $\mathrm{SADABS}^{2}$ was used to perform scaling and absorption corrections. Space group assignments were based on systematic absences, E statistics, and successful refinement of the structures. The structures were solved by direct methods (SHELXS) ${ }^{3}$ and

Bruker SAINT, Bruker-AXS Inc.: Madison, Wisconsin, USA, 2005.

Sheldrick, G. M. SADABS, University of Göttingen, Germany, 2007.

Sheldrick, G. M. Acta Cryst. 1990, A46, 467. 
refined against $\mathrm{F}^{2}$ on all data by full matrix least squares with SHELXL-97. ${ }^{4}$ All non-hydrogen atoms were refined anisotropically; all hydrogen atoms were placed at idealized positions and refined using a riding model.

The structures of $\mathbf{1}$ and $\mathbf{2}$ are identical with the exception of the partially occupied water molecule on a special position in the latter case. The two structures, however, are not strictly isostructural and it is not possible to use one model in a refinement against the other dataset. Although the $x$ and $z$ positions of all atoms in both structures are identical within error, the $y$ coordinate of $\mathrm{Cu}(1)$ differs by 0.5 , and the $y$ coordinates of all other atoms are different. The additional water molecule is located on the crystallographic $\overline{3}$ axis (coordinates $0,0,0$; Wykoff $b$ ); its occupancy was refined freely and eventually set at the convergence value of $0.322 \times$ 0.16667 .

The structure of the $\mathrm{C}_{32} \mathrm{H}_{24} \mathrm{Cu}_{6} \mathrm{O}_{26}$ byproduct (3) contains a 16-membered ring. The structure provides evidence that some 1,3-benzenedicarboxylate is oxidized in the 2-position under the given conditions. Excluding their bond to one another, $\mathrm{Cu}(1)$ and $\mathrm{Cu}(2)$ are each in pseudosquare planar coordination environments, each with two bonds to monodentate carboxylate oxygens and two bonds to the newly formed oxide. The $\mathrm{Cu}(1)-\mathrm{Cu}(2)$ distance is 2.9714(5) $\AA$, which is within bonding distance for two $\mathrm{Cu}(\mathrm{II})$ ions. $\mathrm{Cu}(3)$ is pseudo-square pyramidal; the base of the pyramid contains two bonds to monodentate carboxylate oxygens and two bonds to water molecules, and the apex of the pyramid is a bonded water molecule. Remarkably, in this incomplete octahedral coordination sphere, no residual electron density can be found near the site of the missing ligand. The two $\mathrm{Cu}(3)$ atoms bridge the two $\mathrm{Cu}(1)-\mathrm{Cu}(2)$ dimers in a ring-like structure in the formula unit. The compound crystallizes in the centrosymmetric space group $P-$ 1. The packing diagram viewed along the $c$ axis of the unit cell shows that the 16 -membered ring is not planar and the planes of the dimers are staggered with respect to one another.

4 Sheldrick, G. M. SHELXL 97, University of Göttingen, Germany, 1997. 
Magnetic Measurements. DC magnetic susceptibility data were collected on (ground-up) crystalline samples contained in gelatin capsules using Quantum Design MPMS-5S and MPMSXL SQUID magnetometers at temperatures ranging from 2 to $350 \mathrm{~K}$ and field strengths varying from -50 to $50 \mathrm{kOe}$. The data were corrected for diamagnetic contributions of the sample holder by measurement of an empty capsule, and of the sample itself by use of Pascal's constants.

Other Physical Measurements. Infrared spectra of samples in $\mathrm{KBr}$ pellets were recorded on a Nicolet Magna-IR 860 spectrometer equipped with a KBr beam splitter and a DTGS detector. Powder X-ray diffraction patterns were measured using a Rigaku RU300 rotating anode X-ray diffractometer with $\mathrm{Cu} \mathrm{K \alpha}$ radiation $(\lambda=1.5405 \AA$ ), which was wavelength-selected with a single-crystal graphite monochromator. Samples were spread onto a glass slide fixed with double-sided Scotch tape. Samples were rotated through $2 \theta / \theta$ space and intensity was recorded as a function of $2 \theta$ from 5 to $60^{\circ}$. Patterns were indexed with MDI Jade software version 7.0 and referenced using the JCPDS powder diffraction database. Specific heat measurements were performed using a Quantum Design Model 6000 Physical Properties Measurement System with Helium-3 option. This system uses relaxation method calorimetry and determines specific heat by fitting the temperature response to a two exponential model. A single crystal of mass $0.999 \pm$ $0.002 \mathrm{mg}$ was held to the measurement platform using Apiezon $\mathrm{N}$ grease. The long time constant $\left(\tau_{1}\right)$ varied between 20 and 80 seconds for the temperature range measured. The sample coupling always exceeded $96 \%$, implying that the ratio of the thermal conductance of the grease to that of the platform wires was at least 25. Thermogravimetric analysis measurements were performed using a Seiko Dual TG/DTA 320 Thermogravimetric/Differential Thermal Analyzer (SC). Samples were referenced and measured in aluminum pans, with $\mathrm{N}_{2}$ as a purge gas flowing at 150 $\mathrm{cc} / \mathrm{min}$. Data were recorded at $0.2 \mathrm{~s}$ increments from $20{ }^{\circ} \mathrm{C}$ to $500{ }^{\circ} \mathrm{C}$ at a $5{ }^{\circ} \mathrm{C} / \mathrm{min}$ heating rate. 


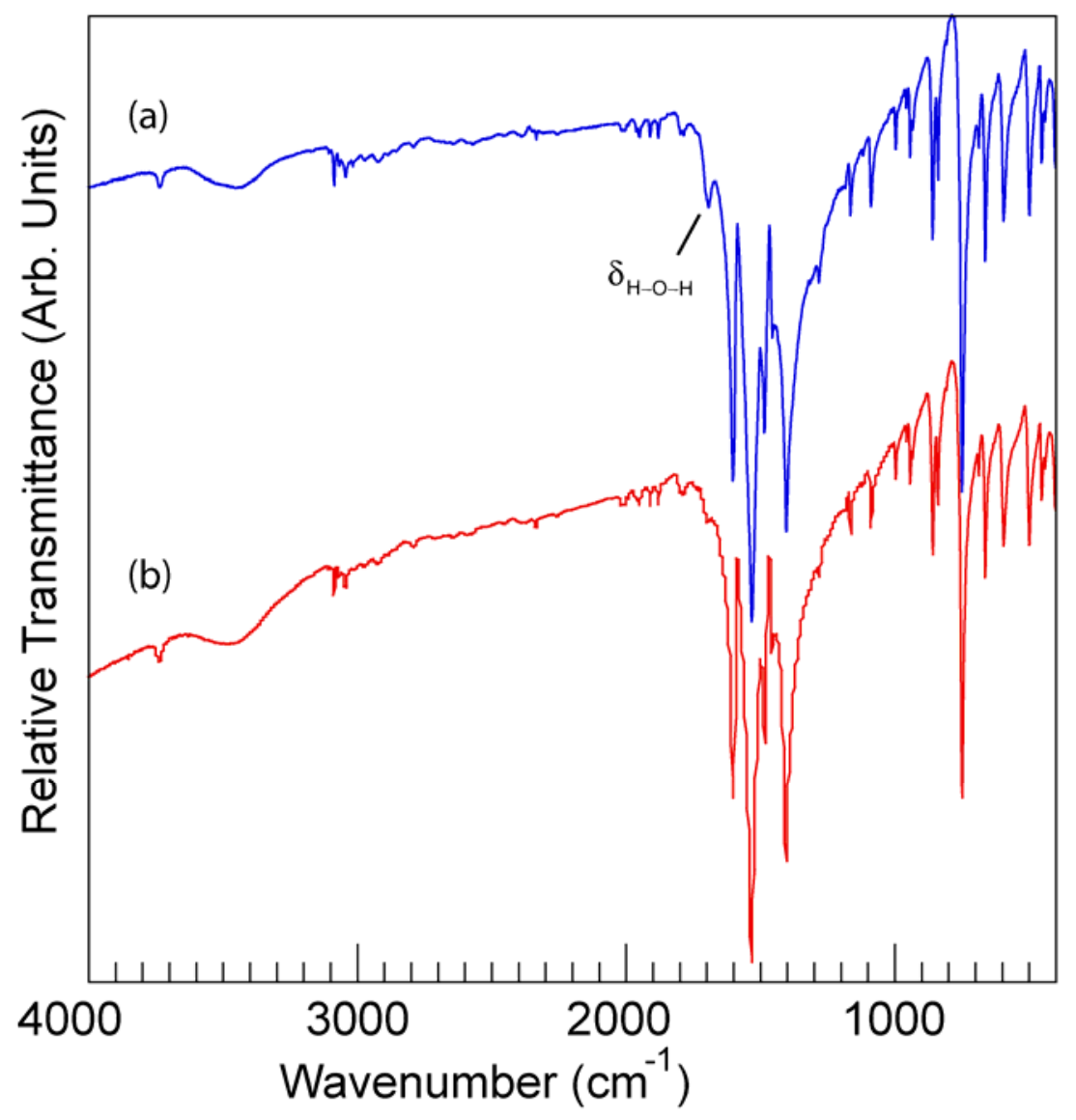

Figure S1. FTIR of a crystalline sample of the title compound (a) before, and (b) after heating at $240{ }^{\circ} \mathrm{C}$ for 2 hours. Before heating, the sample shows an absorbance at $1693 \mathrm{~cm}^{-1}$ due to a bending mode of solvent water. After heating, this absorbance is largely absent. Data for (a): 3736(w), 3086(w), 3043(w), 2016(w), 1950(w), 1909(w), 1880(w), 1795(w), 1693(w), 1603(s), 1531(i, s), 1485(sh), 1456(sh), 1404(i, s), 1282(sh), 1165(s), 1088(s), 997(s), 957(sh), 943(s), 933(sh), 858(s), 839(s), 808(sh), 748(i, s), 687(w), 663(s), 594(s), 548(sh), 499(s), 453(s), $440(\mathrm{sh}), 401(\mathrm{~s}) . \mathrm{s}=$ sharp, $\mathrm{i}=$ intense, $\mathrm{w}=$ weak, $\mathrm{sh}=$ shoulder. 

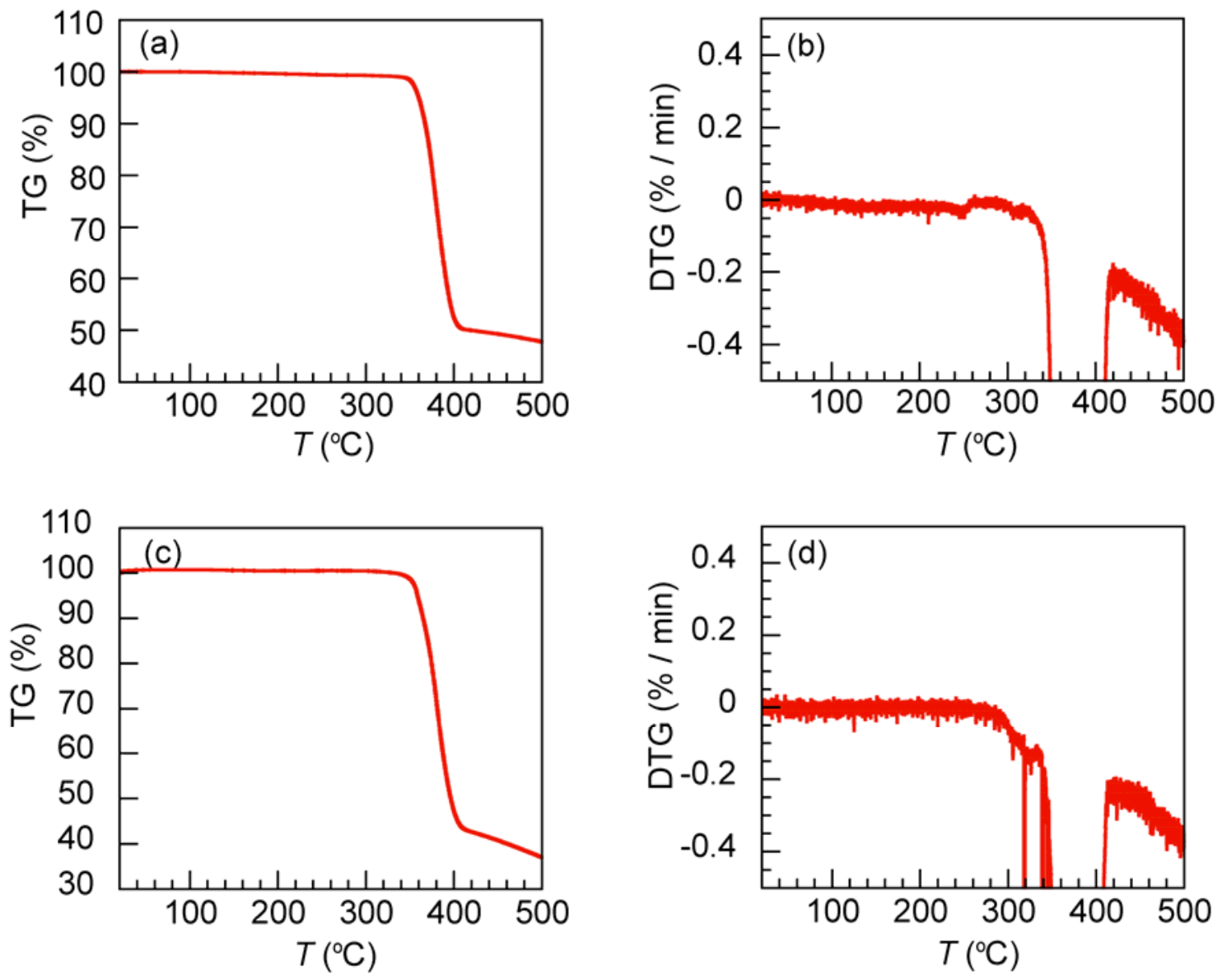

Figure S2. (a) Thermogravimetric analysis for a crystalline sample of the title compound as prepared, showing loss of $0.65 \%$ by mass (or $\sim 0.08 \mathrm{H}_{2} \mathrm{O}$ ) to $257{ }^{\circ} \mathrm{C}$, followed by decomposition of the sample at $300{ }^{\circ} \mathrm{C}$. (b) A differential plot shows the time profile of the loss of mass to 257 ${ }^{\circ} \mathrm{C}$ more clearly. (c) Thermogravimetric analysis for a crystalline sample of the title compound following further sample preparation (heating for 2 hours at $240{ }^{\circ} \mathrm{C}$ ). No significant loss of mass occurs until decomposition of the sample at $292^{\circ} \mathrm{C}$. (d) A differential plot shows the time profile for the loss of mass, with no significant loss occurring to $292{ }^{\circ} \mathrm{C}$. 

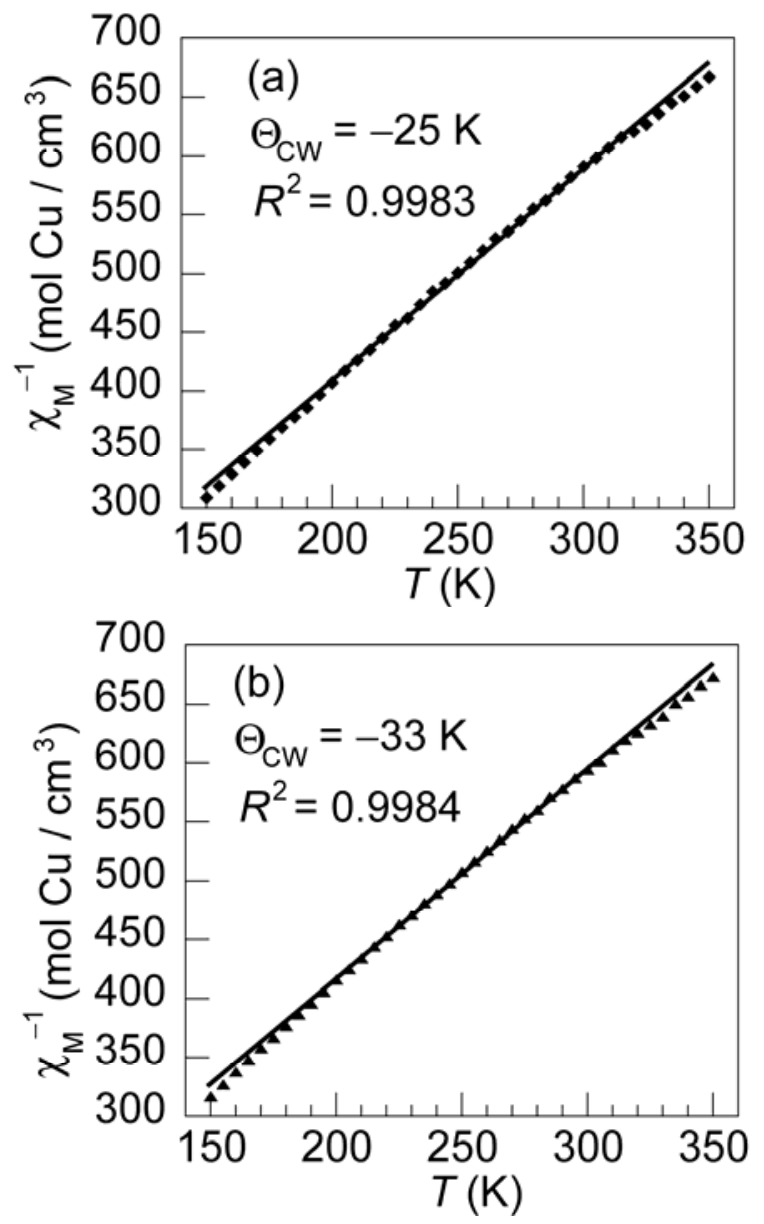

Figure S3. Temperature dependence of $\chi^{-1}$ for a ground sample of the title compound (a) with no heating and (b) after heating crystals to $240{ }^{\circ} \mathrm{C}$ for $2 \mathrm{~h}$ prior to grinding them for SQUID measurement. Values for $\Theta_{\mathrm{CW}}$ were determined by fitting data in the temperature range $150 \leq T \leq$ $350 \mathrm{~K}$. Lines indicate best fit obtained. 


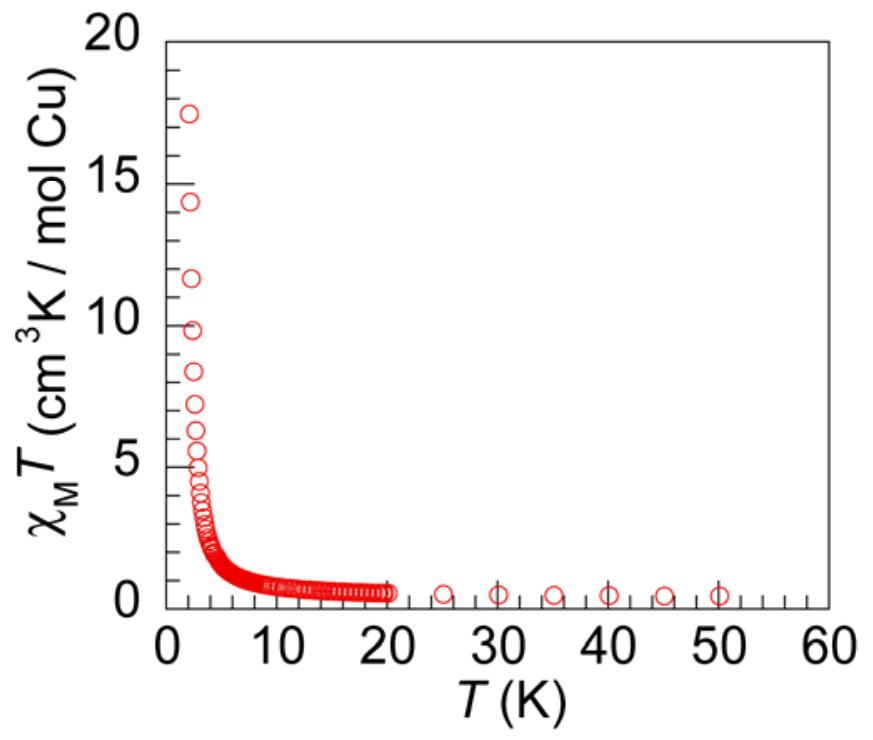

Figure S4. Temperature dependence of $\chi T$ for a ground sample of the title compound after heating crystals to $240{ }^{\circ} \mathrm{C}$ for $2 \mathrm{~h}$ prior to grinding them for SQUID measurement. The data show an upturn at low $T$, indicative of net ferromagnetism. 


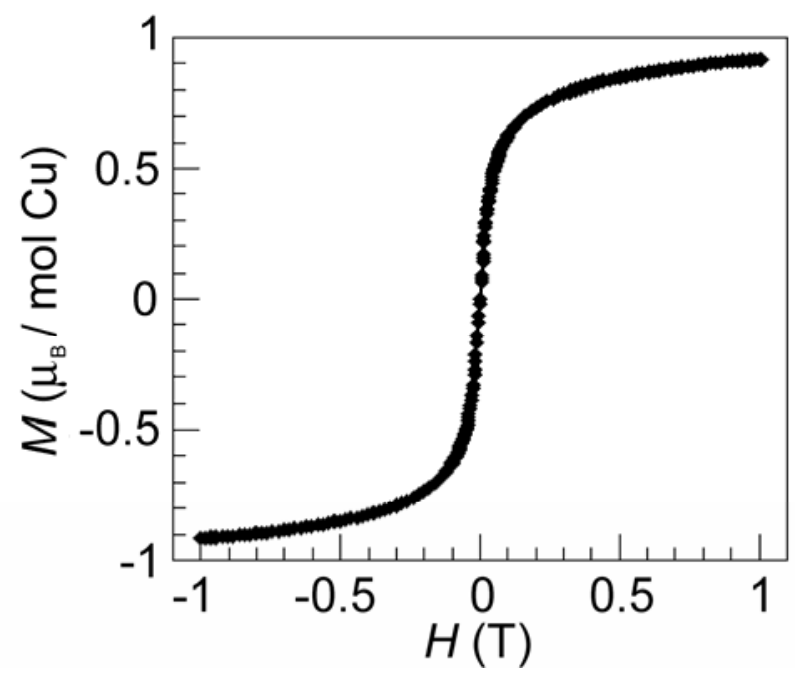

Figure S5. Field dependence of the magnetization per mole of $\mathrm{Cu}$ measured at $2 \mathrm{~K}$. A ground sample of the title compound was measured after heating crystals to $240{ }^{\circ} \mathrm{C}$ for $2 \mathrm{~h}$ prior to grinding them for SQUID measurement. The line is drawn to guide the eye. The magnetization saturates at $0.91 \mu_{\mathrm{B}} / \mathrm{mol} \mathrm{Cu}$, which is consistent with 1 unpaired electron per $\mathrm{Cu}$ center. 


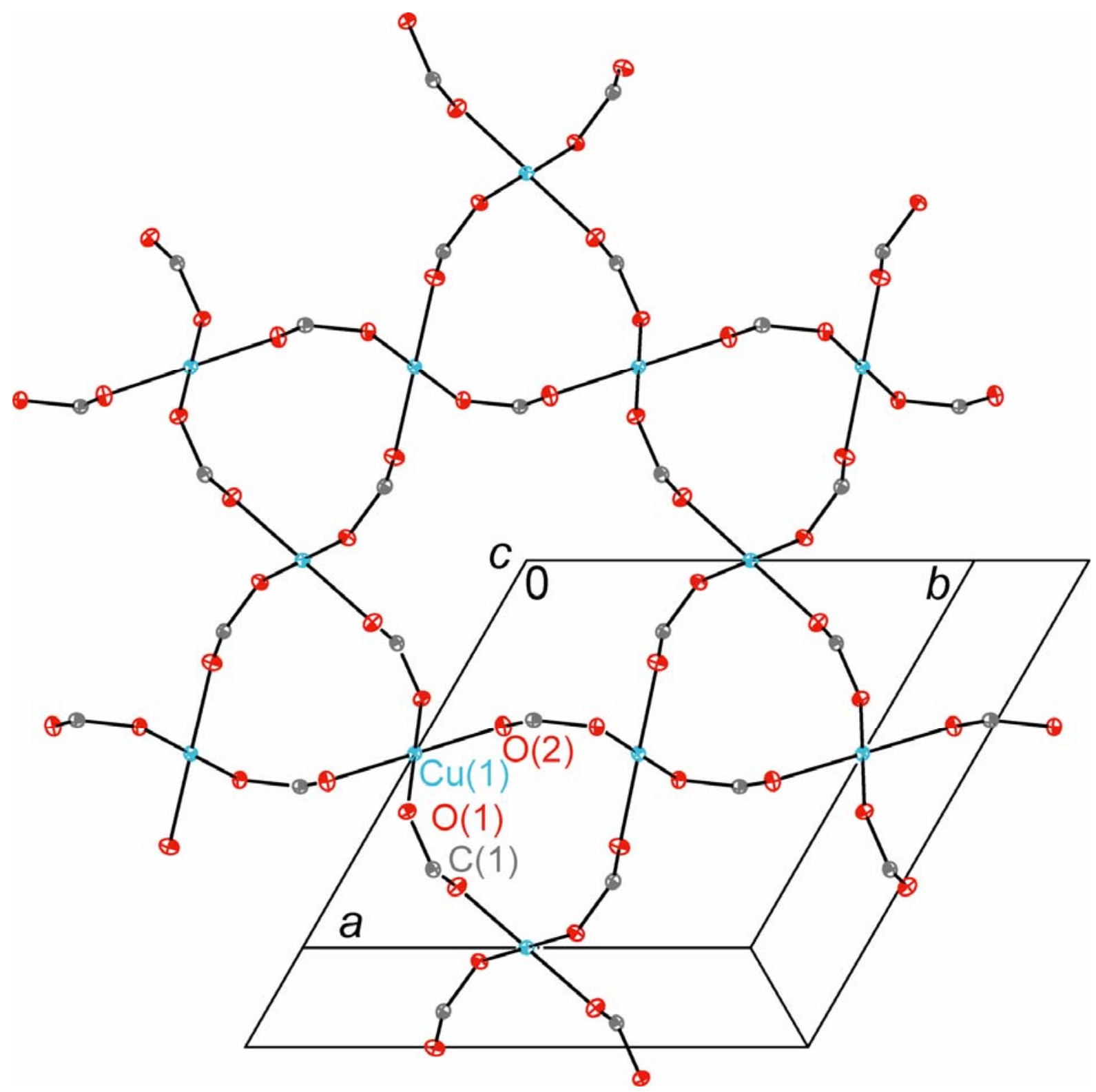

Figure S6. A portion of the crystal structure of $\mathrm{Cu}(1,3-\mathrm{bdc})$, with unit cell. $50 \%$ ellipsoids are shown. Symmetry equivalent atoms (shown but not labeled here) are given in Tables S3 and S4. Phenyl-ring carbon and hydrogen atoms are omitted for clarity. 


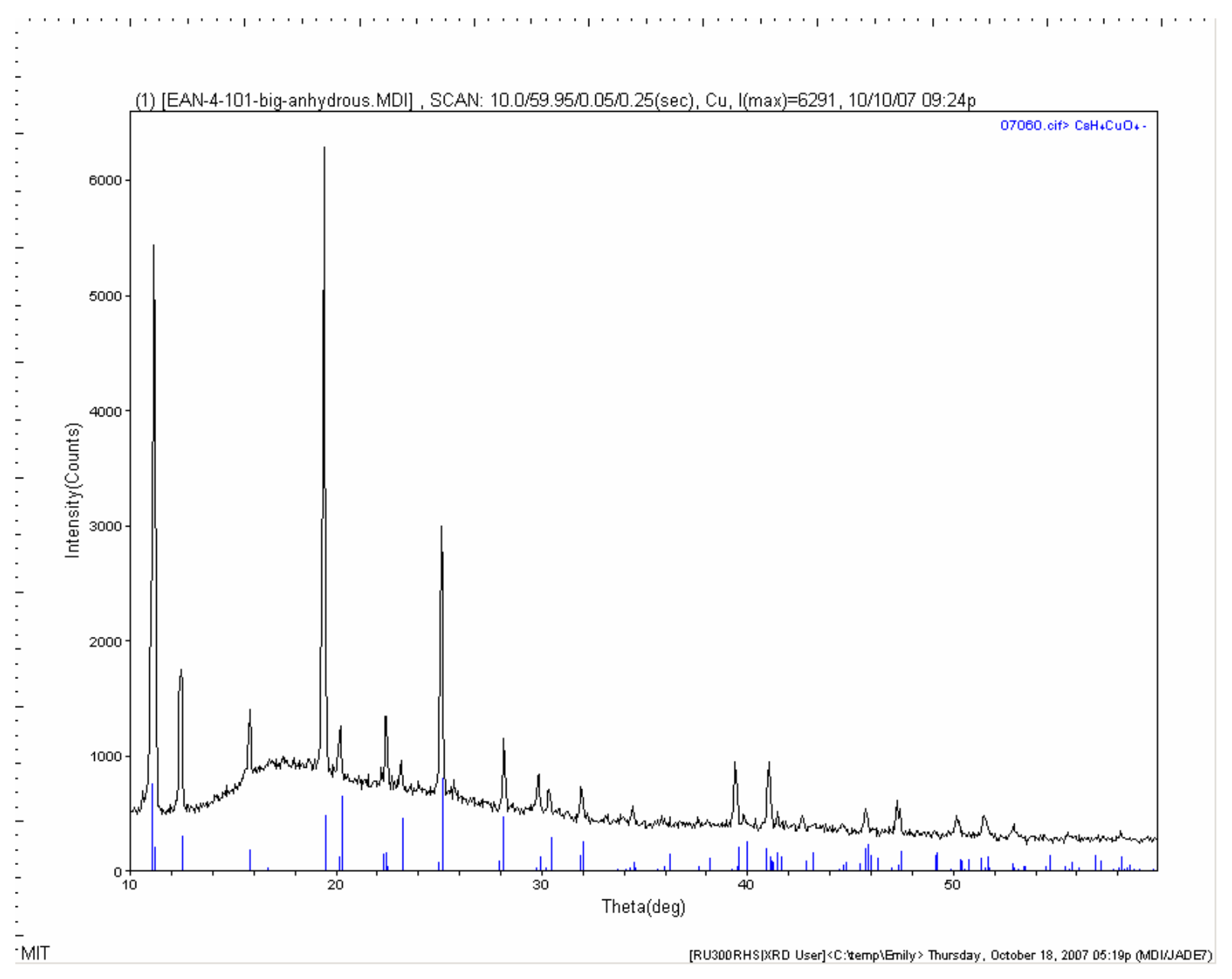

Figure S7. Calculated and observed powder pattern for a crystalline sample of the title compound, after heating for 2 hours at $240{ }^{\circ} \mathrm{C}$. 
Table S1. Crystal data and structure refinement for $\mathrm{Cu}(1,3-\mathrm{bdc})$.

\begin{tabular}{|c|c|c|}
\hline Identification code & 07060 & \\
\hline Empirical formula & $\mathrm{C}_{8} \mathrm{H}_{4} \mathrm{CuO}_{4}$ & \\
\hline Formula weight & 227.65 & \\
\hline Temperature & $100(2) \mathrm{K}$ & \\
\hline Wavelength & $0.71073 \AA$ & \\
\hline Crystal system & Hexagonal & \\
\hline Space group & $P 6_{3} / m$ & \\
\hline \multirow[t]{3}{*}{ Unit cell dimensions } & $a=9.1081(2) \AA$ & $\alpha=90^{\circ}$ \\
\hline & $b=9.1081(2) \AA$ & $\beta=90^{\circ}$ \\
\hline & $c=15.9432(5) \AA$ & $\gamma=120^{\circ}$ \\
\hline Volume, Z & $1145.41(5) \AA^{3}$ & 6 \\
\hline Density (calculated) & $1.980 \mathrm{~g} / \mathrm{cm}^{3}$ & \\
\hline Absorption coefficient & $2.831 \mathrm{~mm}^{-1}$ & \\
\hline $\mathrm{F}(000)$ & 678 & \\
\hline Crystal size & $0.48 \times 0.35 \times 0.15 \mathrm{~mm}$ & \\
\hline$\theta$ range for data collection & 2.56 to $29.57^{\circ}$ & \\
\hline \multirow[t]{3}{*}{ Limiting indices } & $-12 \leq h \leq 12$ & \\
\hline & $-12 \leq k \leq 12$ & \\
\hline & $-22 \leq l \leq 22$ & \\
\hline Reflections collected & 25614 & \\
\hline Independent reflections & $1119\left(\mathrm{R}_{\mathrm{int}}=0.0247\right)$ & \\
\hline Completeness to $\theta=29.57^{\circ}$ & $100.0 \%$ & \\
\hline Refinement method & Full-matrix least-squares on $\mathrm{F}^{2}$ & \\
\hline Data / restraints / parameters & $1119 / 0 / 64$ & \\
\hline Goodness-of-fit on $\mathrm{F}^{2}$ & 1.098 & \\
\hline Final $R$ indices $[\mathrm{I}>2 \sigma(\mathrm{I})]$ & $R_{1}=0.0212$ & $w R_{2}=0.0667$ \\
\hline $\mathrm{R}$ indices (all data) & $R_{1}=0.0217$ & $w R_{2}=0.0674$ \\
\hline Largest diff. peak and hole & $0.528 \mathrm{e}^{-3}$ & $-0.633 \mathrm{e} \AA^{-3}$ \\
\hline
\end{tabular}


Table S2. Atomic coordinates $\left[\times 10^{4}\right]$ and equivalent isotropic displacement parameters $\left[\AA^{2} \times 10^{3}\right]$ for $\mathrm{Cu}(1,3-\mathrm{bdc})$. $U(e q)$ is defined as one third of the trace of the orthogonalized $U_{i j}$ tensor.

\begin{tabular}{lcccr}
\hline & $x$ & $y$ & $z$ & $U(e q)$ \\
\hline $\mathrm{Cu}(1)$ & 5000 & 0 & 0 & $7(1)$ \\
$\mathrm{O}(1)$ & $6360(1)$ & $570(1)$ & $1002(1)$ & $9(1)$ \\
$\mathrm{O}(2)$ & $4262(1)$ & $1614(1)$ & $261(1)$ & $11(1)$ \\
$\mathrm{C}(1)$ & $7828(2)$ & $1867(2)$ & $950(1)$ & $8(1)$ \\
$\mathrm{C}(2)$ & $8839(2)$ & $2404(2)$ & $1741(1)$ & $8(1)$ \\
$\mathrm{C}(3)$ & $10610(2)$ & $3362(2)$ & $1740(1)$ & $10(1)$ \\
$\mathrm{C}(4)$ & $11485(2)$ & $3829(2)$ & 2500 & $11(1)$ \\
$\mathrm{C}(5)$ & $7962(2)$ & $1915(2)$ & 2500 & $8(1)$ \\
\hline
\end{tabular}

Table S3. Bond lengths $[\AA]$ for $\mathrm{Cu}(1,3-\mathrm{bdc})$.

\begin{tabular}{llll}
\hline $\mathrm{Cu}(1)-\mathrm{O}(1)^{a}$ & $1.9265(9)$ & $\mathrm{C}(1)-\mathrm{C}(2)$ & $1.4929(16)$ \\
$\mathrm{Cu}(1)-\mathrm{O}(1)$ & $1.9265(9)$ & $\mathrm{C}(2)-\mathrm{C}(5)$ & $1.3948(14)$ \\
$\mathrm{Cu}(1)-\mathrm{O}(2)^{a}$ & $1.9428(9)$ & $\mathrm{C}(2)-\mathrm{C}(3)$ & $1.3985(16)$ \\
$\mathrm{Cu}(1)-\mathrm{O}(2)$ & $1.9428(9)$ & $\mathrm{C}(3)-\mathrm{C}(4)$ & $1.3949(14)$ \\
$\mathrm{O}(1)-\mathrm{C}(1)$ & $1.2685(15)$ & $\mathrm{C}(4)-\mathrm{C}(3)^{d}$ & $1.3949(14)$ \\
$\mathrm{O}(2)-\mathrm{C}(1)^{b}$ & $1.2680(14)$ & $\mathrm{C}(5)-\mathrm{C}(2)^{d}$ & $1.3948(14)$ \\
$\mathrm{C}(1)-\mathrm{O}(2)^{c}$ & $1.2680(14)$ & & \\
\hline
\end{tabular}

Symmetry transformations used to generate equivalent atoms:
${ }^{a}-x+1,-y,-z$
${ }^{b}-x+y+1,-x+1, z$
${ }^{c}-y+1, x-y, z$
${ }^{d} X, y,-z+1 / 2$ 
Table S4. Bond angles $\left[{ }^{\circ}\right]$ for $\mathrm{Cu}(1,3-b d c)$.

\begin{tabular}{llll}
\hline $\mathrm{O}(1)^{a}-\mathrm{Cu}(1)-\mathrm{O}(1)$ & $180.00(7)$ & $\mathrm{O}(2)^{c}-\mathrm{C}(1)-\mathrm{C}(2)$ & $122.61(11)$ \\
$\mathrm{O}(1)^{a}-\mathrm{Cu}(1)-\mathrm{O}(2)^{a}$ & $92.14(4)$ & $\mathrm{O}(1)-\mathrm{C}(1)-\mathrm{C}(2)$ & $116.13(10)$ \\
$\mathrm{O}(1)-\mathrm{Cu}(1)-\mathrm{O}(2)^{a}$ & $87.86(4)$ & $\mathrm{C}(5)-\mathrm{C}(2)-\mathrm{C}(3)$ & $119.90(12)$ \\
$\mathrm{O}(1)^{a}-\mathrm{Cu}(1)-\mathrm{O}(2)$ & $87.86(4)$ & $\mathrm{C}(5)-\mathrm{C}(2)-\mathrm{C}(1)$ & $117.85(11)$ \\
$\mathrm{O}(1)-\mathrm{Cu}(1)-\mathrm{O}(2)$ & $92.14(4)$ & $\mathrm{C}(3)-\mathrm{C}(2)-\mathrm{C}(1)$ & $122.25(11)$ \\
$\mathrm{O}(2)^{a}-\mathrm{Cu}(1)-\mathrm{O}(2)$ & $180.00(7)$ & $\mathrm{C}(4)-\mathrm{C}(3)-\mathrm{C}(2)$ & $119.60(12)$ \\
$\mathrm{C}(1)-\mathrm{O}(1)-\mathrm{Cu}(1)$ & $115.67(8)$ & $\mathrm{C}(3)-\mathrm{C}(4)-\mathrm{C}(3)^{d}$ & $120.64(16)$ \\
$\mathrm{C}(1)^{b}-\mathrm{O}(2)-\mathrm{Cu}(1)$ & $132.42(8)$ & $\mathrm{C}(2)^{d}-\mathrm{C}(5)-\mathrm{C}(2)$ & $120.34(15)$ \\
$\mathrm{O}(2)^{c}-\mathrm{C}(1)-\mathrm{O}(1)$ & $121.26(11)$ & & \\
\hline
\end{tabular}

Symmetry transformations used to generate equivalent atoms:

${ }_{a}-x+1,-y,-z \quad{ }^{b}-x+y+1,-x+1, z \quad{ }^{c}-y+1, x-y, z \quad{ }^{d} x, y,-z+1 / 2$ 
Table S5. Anisotropic displacement parameters $\left[\AA^{2} \times 10^{3}\right]$ for $\mathrm{Cu}(1,3-b d c)$. The anisotropic displacement factor exponent takes the form: $-2 \pi^{2}\left[\left(h a^{*}\right)^{2} U_{11}+\ldots+2 h k a^{*} b^{*} U_{12}\right]$.

\begin{tabular}{lrrrrrr}
\hline & $U_{11}$ & $U_{22}$ & $U_{33}$ & $U_{23}$ & $U_{13}$ & $U_{12}$ \\
\hline $\mathrm{Cu}(1)$ & $8(1)$ & $7(1)$ & $4(1)$ & $-1(1)$ & $-1(1)$ & $4(1)$ \\
$\mathrm{O}(1)$ & $9(1)$ & $10(1)$ & $7(1)$ & $-1(1)$ & $-1(1)$ & $3(1)$ \\
$\mathrm{O}(2)$ & $16(1)$ & $13(1)$ & $6(1)$ & $-1(1)$ & $0(1)$ & $9(1)$ \\
$\mathrm{C}(1)$ & $10(1)$ & $9(1)$ & $6(1)$ & $-1(1)$ & $0(1)$ & $5(1)$ \\
$\mathrm{C}(2)$ & $9(1)$ & $9(1)$ & $6(1)$ & $-1(1)$ & $-1(1)$ & $4(1)$ \\
$\mathrm{C}(3)$ & $9(1)$ & $12(1)$ & $8(1)$ & $1(1)$ & $1(1)$ & $5(1)$ \\
$\mathrm{C}(4)$ & $8(1)$ & $13(1)$ & $11(1)$ & 0 & 0 & $5(1)$ \\
$\mathrm{C}(5)$ & $8(1)$ & $8(1)$ & $7(1)$ & 0 & 0 & $3(1)$ \\
\hline
\end{tabular}

Table S6. Hydrogen coordinates $\left[\times 10^{4}\right]$ and isotropic displacement parameters $\left[\AA^{2} \times 10^{3}\right]$ for $\mathrm{Cu}(1,3-\mathrm{bdc})$.

\begin{tabular}{ccccc}
\hline & $x$ & $y$ & $z$ & $U($ eq $)$ \\
\hline $\mathrm{H}(3)$ & 11214 & 3693 & 1224 & 12 \\
$\mathrm{H}(4)$ & 12689 & 4472 & 2500 & 13 \\
$\mathrm{H}(5)$ & 6760 & 1246 & 2500 & 10 \\
\hline
\end{tabular}




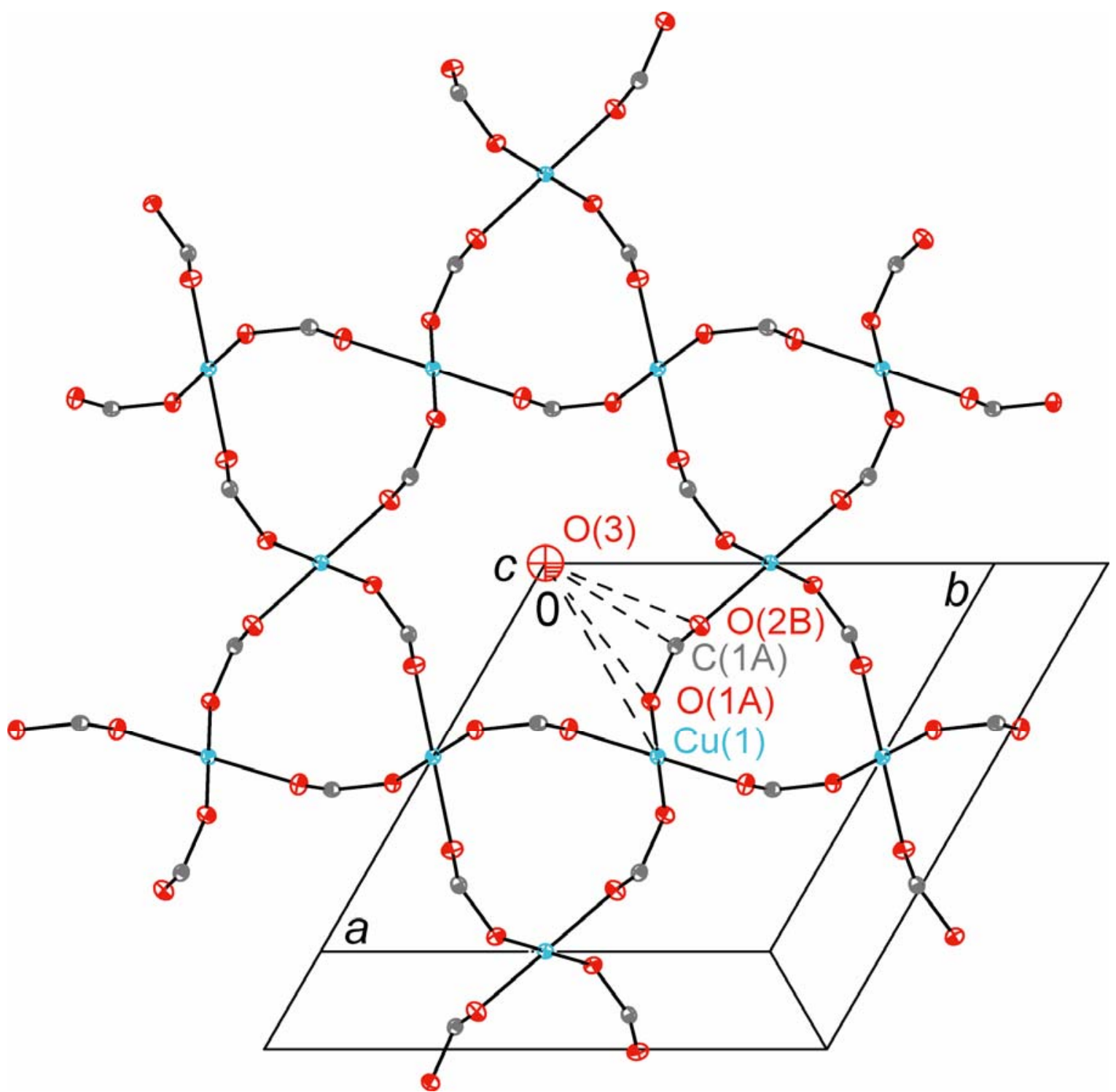

Figure S8. A portion of the crystal structure of $\mathrm{Cu}(1,3-\mathrm{bdc}) \cdot 0.11 \mathrm{H}_{2} \mathrm{O}$, with unit cell. $50 \%$ ellipsoids are shown. $\mathrm{O}(3)$ is the oxygen atom of a solvated water molecule statistically distributed over 1/9 of sites. Symmetry equivalent atoms (shown but not labeled here) are given in Tables S9 and S10. Phenyl-ring carbon atoms and all hydrogen atoms are omitted for clarity. The distances between $\mathrm{O}(3)$ and other atoms in the structure are as follows: $\mathrm{O}(3)-\mathrm{Cu}(1)$, 4.5528(2) Å. $\mathrm{O}(3)-\mathrm{O}(1 \mathrm{~A}), 3.9405(8) \AA . \mathrm{O}(3)-\mathrm{C}(1 \mathrm{~A}), 3.5303(11) \AA ̊ . \mathrm{O}(3)-\mathrm{O}(2 \mathrm{~B}), 3.4166(8) \AA$. 
Table S7. Crystal data and structure refinement for $\mathrm{Cu}(1,3-\mathrm{bdc}) \cdot 0.11 \mathrm{H}_{2} \mathrm{O}$.

\begin{tabular}{|c|c|c|}
\hline Identification code & 05251 & \\
\hline Empirical formula & $\mathrm{C}_{8} \mathrm{H}_{4} \mathrm{CuO}_{4.11}$ & \\
\hline Formula weight & 229.44 & \\
\hline Temperature & $100(2) \mathrm{K}$ & \\
\hline Wavelength & $0.71073 \AA$ & \\
\hline Crystal system & Hexagonal & \\
\hline Space group & $P 6_{3} / m$ & \\
\hline \multirow[t]{3}{*}{ Unit cell dimensions } & $a=9.1056(3) \AA$ & $\alpha=90^{\circ}$ \\
\hline & $b=9.1056(3) \AA$ & $\beta=90^{\circ}$ \\
\hline & $c=15.9550(12) \AA$ & $\gamma=120^{\circ}$ \\
\hline Volume, Z & $1145.63(10) \AA^{3}$ & 6 \\
\hline Density (calculated) & $1.995 \mathrm{~g} / \mathrm{cm}^{3}$ & \\
\hline Absorption coefficient & $2.832 \mathrm{~mm}^{-1}$ & \\
\hline $\mathrm{F}(000)$ & 683 & \\
\hline Crystal size & $0.15 \times 0.10 \times 0.10 \mathrm{~mm}$ & \\
\hline$\theta$ range for data collection & 2.55 to $29.55^{\circ}$ & \\
\hline \multirow[t]{3}{*}{ Limiting indices } & $-12 \leq h \leq 12$ & \\
\hline & $-12 \leq k \leq 12$ & \\
\hline & $-22 \leq l \leq 22$ & \\
\hline Reflections collected & 22913 & \\
\hline Independent reflections & $1118\left(\mathrm{R}_{\mathrm{int}}=0.0311\right)$ & \\
\hline Completeness to $\theta=29.55^{\circ}$ & $100.0 \%$ & \\
\hline Refinement method & Full-matrix least-squares on $\mathrm{F}^{2}$ & \\
\hline Data / restraints / parameters & $1118 / 0 / 66$ & \\
\hline Goodness-of-fit on $\mathrm{F}^{2}$ & 1.101 & \\
\hline Final $R$ indices $[I>2 \sigma(I)]$ & $R_{1}=0.0217$ & $w R_{2}=0.0621$ \\
\hline $\mathrm{R}$ indices (all data) & $R_{1}=0.0235$ & $w R_{2}=0.0637$ \\
\hline Largest diff. peak and hole & $0.578 \mathrm{e}^{-3}$ & $-1.128 \mathrm{e} \AA^{-3}$ \\
\hline
\end{tabular}


Table S8. Atomic coordinates $\left[\times 10^{4}\right]$ and equivalent isotropic displacement parameters $\left[\AA^{2} \times 10^{3}\right]$ for $\mathrm{Cu}(1,3-b d c) \cdot 0.11 \mathrm{H}_{2} \mathrm{O}$. $U(e q)$ is defined as one third of the trace of the orthogonalized $U_{i j}$ tensor.

\begin{tabular}{lcccr}
\hline & $x$ & $y$ & $z$ & $U(e q)$ \\
\hline $\mathrm{Cu}(1)$ & 5000 & 5000 & 0 & $8(1)$ \\
$\mathrm{O}(1)$ & $6361(1)$ & $5790(1)$ & $1002(1)$ & $11(1)$ \\
$\mathrm{O}(2)$ & $4259(1)$ & $2646(1)$ & $261(1)$ & $13(1)$ \\
$\mathrm{C}(1)$ & $7826(1)$ & $5961(1)$ & $950(1)$ & $10(1)$ \\
$\mathrm{C}(2)$ & $8838(1)$ & $6435(1)$ & $1741(1)$ & $10(1)$ \\
$\mathrm{C}(3)$ & $10612(1)$ & $7250(1)$ & $1741(1)$ & $12(1)$ \\
$\mathrm{C}(4)$ & $11484(2)$ & $7656(2)$ & 2500 & $12(1)$ \\
$\mathrm{C}(5)$ & $7963(2)$ & $6049(2)$ & 2500 & $10(1)$ \\
$\mathrm{O}(3)$ & 0 & 0 & 0 & $61(4)$ \\
\hline
\end{tabular}

Table S9. Bond lengths $[\AA]$ for $\mathrm{Cu}(1,3-\mathrm{bdc}) \cdot 0.11 \mathrm{H}_{2} \mathrm{O}$.

\begin{tabular}{llll}
\hline $\mathrm{Cu}(1)-\mathrm{O}(1)^{a}$ & $1.9275(8)$ & $\mathrm{C}(1)-\mathrm{C}(2)$ & $1.4932(15)$ \\
$\mathrm{Cu}(1)-\mathrm{O}(1)$ & $1.9275(8)$ & $\mathrm{C}(2)-\mathrm{C}(5)$ & $1.3940(13)$ \\
$\mathrm{Cu}(1)-\mathrm{O}(2)$ & $1.9437(8)$ & $\mathrm{C}(2)-\mathrm{C}(3)$ & $1.4006(16)$ \\
$\mathrm{Cu}(1)-\mathrm{O}(2)^{a}$ & $1.9437(8)$ & $\mathrm{C}(3)-\mathrm{C}(4)$ & $1.3934(14)$ \\
$\mathrm{O}(1)-\mathrm{C}(1)$ & $1.2665(14)$ & $\mathrm{C}(4)-\mathrm{C}(3)^{d}$ & $1.3934(14)$ \\
$\mathrm{O}(2)-\mathrm{C}(1)^{b}$ & $1.2692(14)$ & $\mathrm{C}(5)-\mathrm{C}(2)^{d}$ & $1.3940(13)$ \\
$\mathrm{C}(1)-\mathrm{O}(2)^{c}$ & $1.2692(14)$ & & \\
\hline
\end{tabular}

Symmetry transformations used to generate equivalent atoms:

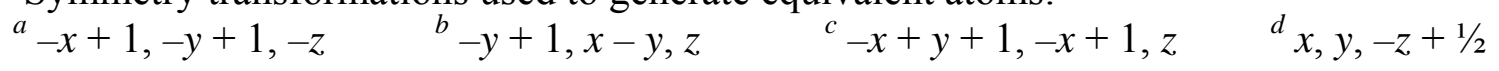


Table S10. Bond angles $\left[^{\circ}\right]$ for $\mathrm{Cu}(1,3-\mathrm{bdc}) \cdot 0.11 \mathrm{H}_{2} \mathrm{O}$.

\begin{tabular}{llll}
\hline $\mathrm{O}(1)^{a}-\mathrm{Cu}(1)-\mathrm{O}(1)$ & $180.00(3)$ & $\mathrm{O}(1)-\mathrm{C}(1)-\mathrm{C}(2)$ & $116.12(10)$ \\
$\mathrm{O}(1)^{a}-\mathrm{Cu}(1)-\mathrm{O}(2)$ & $87.84(3)$ & $\mathrm{O}(2)^{c}-\mathrm{C}(1)-\mathrm{C}(2)$ & $122.63(10)$ \\
$\mathrm{O}(1)-\mathrm{Cu}(1)-\mathrm{O}(2)$ & $92.16(3)$ & $\mathrm{C}(5)-\mathrm{C}(2)-\mathrm{C}(3)$ & $119.78(11)$ \\
$\mathrm{O}(1)^{a}-\mathrm{Cu}(1)-\mathrm{O}(2)^{a}$ & $92.16(3)$ & $\mathrm{C}(5)-\mathrm{C}(2)-\mathrm{C}(1)$ & $117.97(10)$ \\
$\mathrm{O}(1)-\mathrm{Cu}(1)-\mathrm{O}(2)^{a}$ & $87.84(3)$ & $\mathrm{C}(3)-\mathrm{C}(2)-\mathrm{C}(1)$ & $122.25(10)$ \\
$\mathrm{O}(2)-\mathrm{Cu}(1)-\mathrm{O}(2)^{a}$ & $180.00(5)$ & $\mathrm{C}(4)-\mathrm{C}(3)-\mathrm{C}(2)$ & $119.55(11)$ \\
$\mathrm{C}(1)-\mathrm{O}(1)-\mathrm{Cu}(1)$ & $115.69(7)$ & $\mathrm{C}(3)-\mathrm{C}(4)-\mathrm{C}(3)^{d}$ & $120.81(15)$ \\
$\mathrm{C}(1)^{b}-\mathrm{O}(2)-\mathrm{Cu}(1)$ & $132.36(8)$ & $\mathrm{C}(2)^{d}-\mathrm{C}(5)-\mathrm{C}(2)$ & $120.53(14)$ \\
$\mathrm{O}(1)-\mathrm{C}(1)-\mathrm{O}(2)^{c}$ & $121.25(10)$ & & \\
\hline
\end{tabular}

Symmetry transformations used to generate equivalent atoms:

${ }_{a}^{a}-x+1,-y+1,-z \quad b_{-y+1, x-y, z}{ }^{c}-x+y+1,-x+1, z \quad{ }^{d} x, y,-z+1 / 2$ 
Table S11. Anisotropic displacement parameters $\left[\AA^{2} \times 10^{3}\right]$ for $\mathrm{Cu}(1,3-b d c) \cdot 0.11 \mathrm{H}_{2} \mathrm{O}$. The anisotropic displacement factor exponent takes the form: $-2 \pi^{2}\left[\left(h a^{*}\right)^{2} U_{11}+\ldots+2 h k a^{*} b^{*} U_{12}\right]$.

\begin{tabular}{lrrrrrr}
\hline & $U_{11}$ & $U_{22}$ & $U_{33}$ & $U_{23}$ & $U_{13}$ & $U_{12}$ \\
\hline $\mathrm{Cu}(1)$ & $10(1)$ & $10(1)$ & $6(1)$ & $0(1)$ & $-1(1)$ & $5(1)$ \\
$\mathrm{O}(1)$ & $12(1)$ & $14(1)$ & $9(1)$ & $0(1)$ & $-1(1)$ & $7(1)$ \\
$\mathrm{O}(2)$ & $19(1)$ & $12(1)$ & $8(1)$ & $0(1)$ & $-1(1)$ & $8(1)$ \\
$\mathrm{C}(1)$ & $11(1)$ & $9(1)$ & $9(1)$ & $1(1)$ & $0(1)$ & $5(1)$ \\
$\mathrm{C}(2)$ & $12(1)$ & $11(1)$ & $8(1)$ & $0(1)$ & $-1(1)$ & $6(1)$ \\
$\mathrm{C}(3)$ & $11(1)$ & $12(1)$ & $11(1)$ & $0(1)$ & $2(1)$ & $5(1)$ \\
$\mathrm{C}(4)$ & $9(1)$ & $13(1)$ & $13(1)$ & 0 & 0 & $3(1)$ \\
$\mathrm{C}(5)$ & $10(1)$ & $10(1)$ & $10(1)$ & 0 & 0 & $5(1)$ \\
$\mathrm{O}(3)$ & $54(6)$ & $54(6)$ & $75(12)$ & 0 & 0 & $27(3)$ \\
\hline
\end{tabular}

Table S12. Hydrogen coordinates $\left[\times 10^{4}\right]$ and isotropic displacement parameters $\left[\AA^{2} \times 10^{3}\right]$ for $\mathrm{Cu}\left(1,3\right.$-bdc) $0.11 \mathrm{H}_{2} \mathrm{O}$.

\begin{tabular}{ccccc}
\hline & $x$ & $y$ & $z$ & $U(e q)$ \\
\hline $\mathrm{H}(3)$ & 11216 & 7523 & 1226 & 14 \\
$\mathrm{H}(4)$ & 12687 & 8216 & 2500 & 15 \\
$\mathrm{H}(5)$ & 6761 & 5519 & 2500 & 12 \\
\hline
\end{tabular}




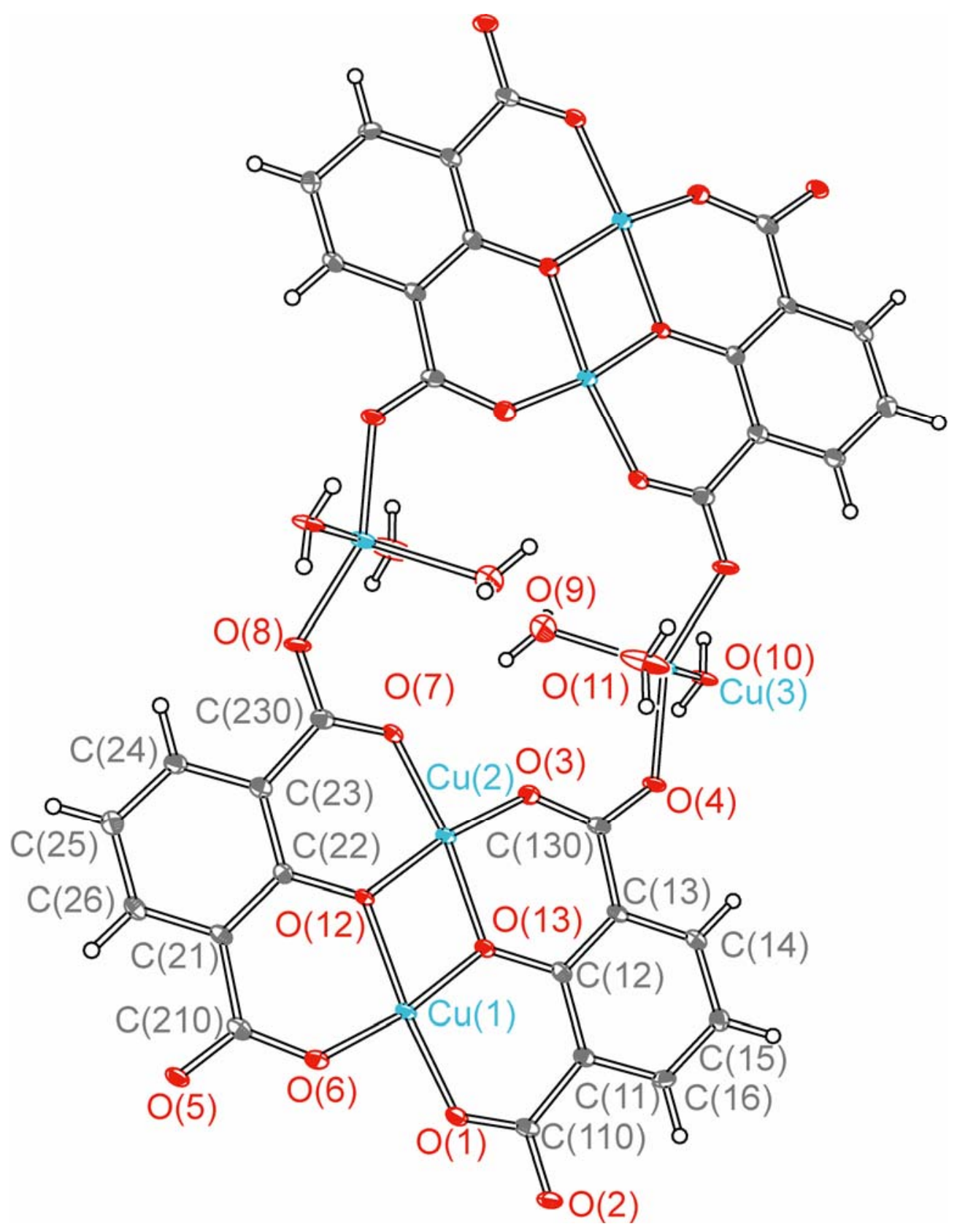

Figure S9. A portion of the crystal structure of $\mathrm{C}_{32} \mathrm{H}_{24} \mathrm{Cu}_{6} \mathrm{O}_{26} .50 \%$ ellipsoids are shown. Symmetry equivalent atoms (shown but not labeled here) are given in Tables S15 and S16. 


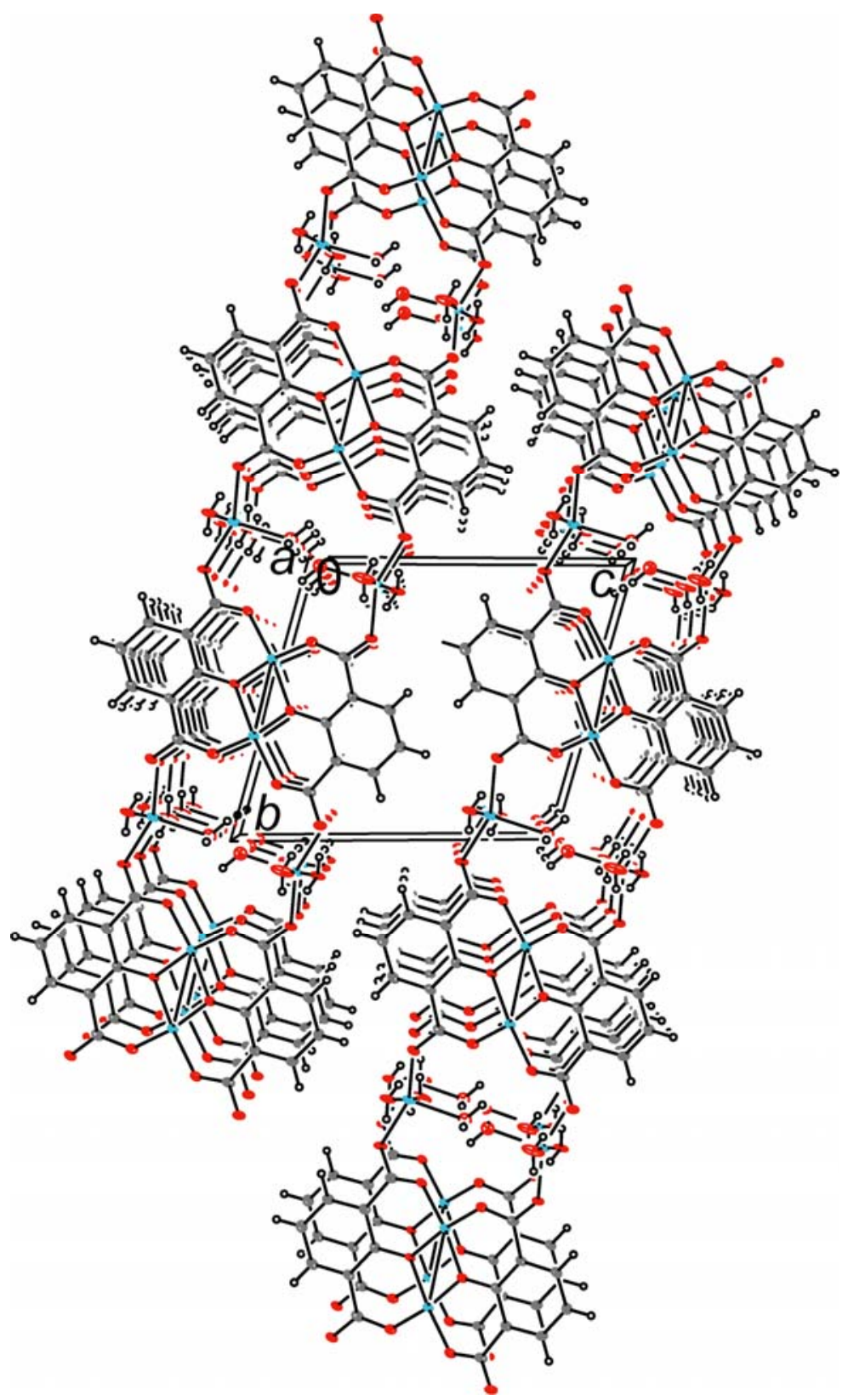

Figure S10. Packing diagram for $\mathrm{C}_{32} \mathrm{H}_{24} \mathrm{Cu}_{6} \mathrm{O}_{26}$, as viewed down the crystallographic $a$ axis. $50 \%$ thermal ellipsoids are shown. $\mathrm{Cu}$ atoms are shown in teal, $\mathrm{O}$ atoms in red, $\mathrm{C}$ atoms in gray, and $\mathrm{H}$ atoms in white. 


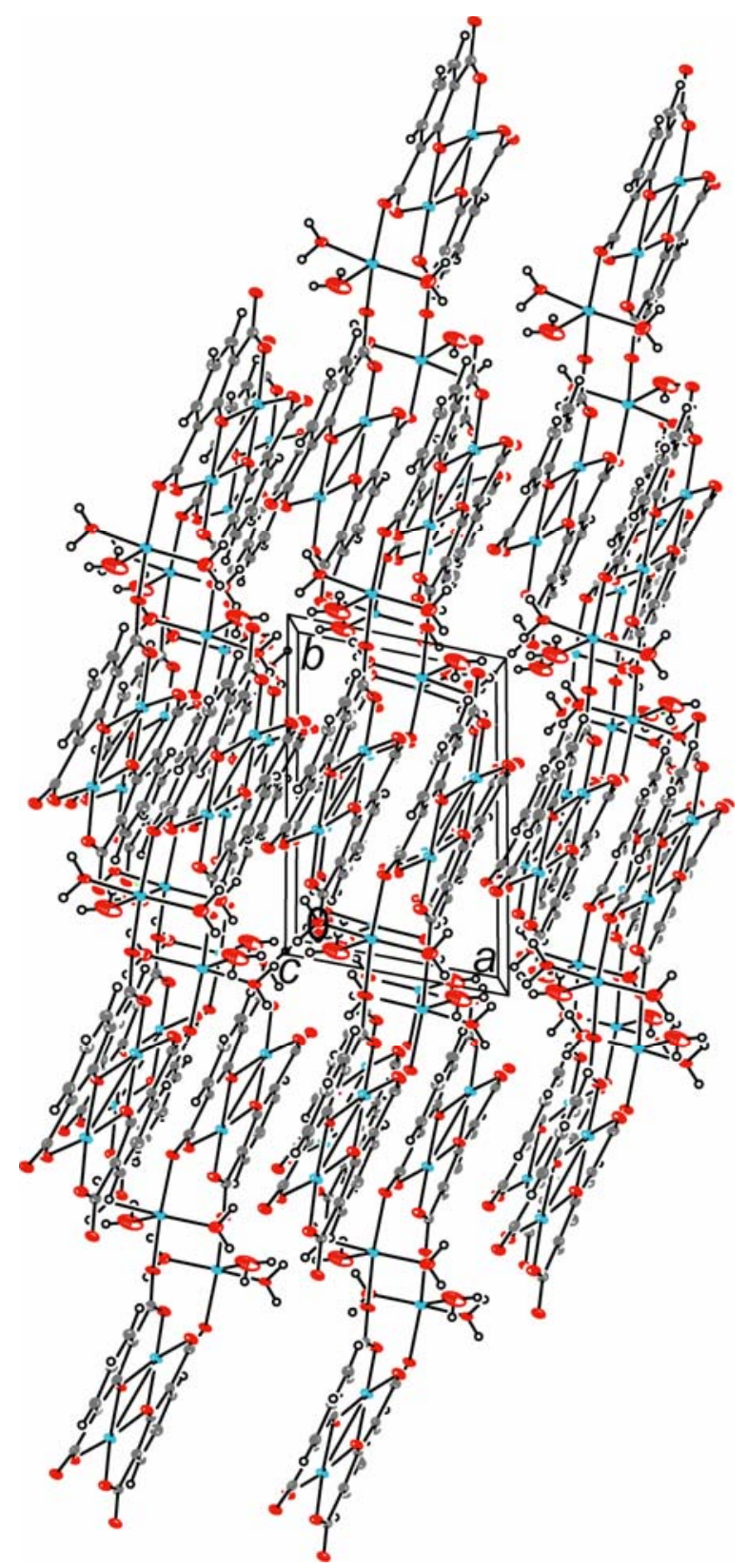

Figure S11. Packing diagram for $\mathrm{C}_{32} \mathrm{H}_{24} \mathrm{Cu}_{6} \mathrm{O}_{26}$, as viewed down the crystallographic $c$ axis. $50 \%$ thermal ellipsoids are shown. $\mathrm{Cu}$ atoms are shown in teal, $\mathrm{O}$ atoms in red, $\mathrm{C}$ atoms in gray, and $\mathrm{H}$ atoms in white. 
Table S13. Crystal data and structure refinement for $\mathrm{C}_{32} \mathrm{H}_{24} \mathrm{Cu}_{6} \mathrm{O}_{26}$.

\begin{tabular}{|c|c|c|}
\hline Identification code & 06218 & \\
\hline Empirical formula & $\mathrm{C}_{32} \mathrm{H}_{24} \mathrm{Cu}_{6} \mathrm{O}_{26}$ & \\
\hline Formula weight & 1205.75 & \\
\hline Temperature & $100(2) \mathrm{K}$ & \\
\hline Wavelength & $0.71073 \AA$ & \\
\hline Crystal system & Triclinic & \\
\hline Space group & $P-1$ & \\
\hline \multirow[t]{3}{*}{ Unit cell dimensions } & $a=7.0738(6) \AA$ & $\alpha=104.0140(10)^{\circ}$ \\
\hline & $b=10.8611(8) \AA$ & $\beta=99.4120(10)^{\circ}$ \\
\hline & $c=12.0790(9) \AA$ & $\gamma=98.2080(10)^{\circ}$ \\
\hline Volume, Z & $872.01(12) \AA^{3}$ & 1 \\
\hline Density (calculated) & $2.296 \mathrm{~g} / \mathrm{cm}^{3}$ & \\
\hline Absorption coefficient & $3.705 \mathrm{~mm}^{-1}$ & \\
\hline $\mathrm{F}(000)$ & 598 & \\
\hline Crystal size & $0.20 \times 0.10 \times 0.02 \mathrm{~mm}$ & \\
\hline$\theta$ range for data collection & 1.97 to $29.13^{\circ}$ & \\
\hline \multirow[t]{3}{*}{ Limiting indices } & $-9 \leq h \leq 9$ & \\
\hline & $-14 \leq k \leq 14$ & \\
\hline & $-16 \leq l \leq 16$ & \\
\hline Reflections collected & 18271 & \\
\hline Independent reflections & $4692\left(\mathrm{R}_{\mathrm{int}}=0.0450\right)$ & \\
\hline Completeness to $\theta=29.13^{\circ}$ & $99.7 \%$ & \\
\hline Refinement method & Full-matrix least-squares on $\mathrm{F}^{2}$ & \\
\hline Data / restraints / parameters & 4692 / 9 / 307 & \\
\hline Goodness-of-fit on $\mathrm{F}^{2}$ & 1.021 & \\
\hline Final $R$ indices $[\mathrm{I}>2 \sigma(\mathrm{I})]$ & $R_{1}=0.0333$ & $w R_{2}=0.0699$ \\
\hline $\mathrm{R}$ indices (all data) & $R_{1}=0.0512$ & $w R_{2}=0.0768$ \\
\hline Largest diff. peak and hole & $0.689 \mathrm{e}^{-3}$ & $-0.540 \mathrm{e} \AA^{-3}$ \\
\hline
\end{tabular}


Table S14. Atomic coordinates $\left[\times 10^{4}\right]$ and equivalent isotropic displacement parameters $\left[\AA^{2} \times 10^{3}\right]$ for $\mathrm{C}_{32} \mathrm{H}_{24} \mathrm{Cu}_{6} \mathrm{O}_{26}$. U(eq) is defined as one third of the trace of the orthogonalized $U_{i j}$ tensor.

\begin{tabular}{|c|c|c|c|c|}
\hline & $x$ & $y$ & $z$ & $U(e q)$ \\
\hline $\mathrm{Cu}(1)$ & $6472(1)$ & $3610(1)$ & $-80(1)$ & $10(1)$ \\
\hline $\mathrm{Cu}(2)$ & $8589(1)$ & $6226(1)$ & $50(1)$ & $10(1)$ \\
\hline $\mathrm{Cu}(3)$ & $3842(1)$ & $996(1)$ & $2305(1)$ & $13(1)$ \\
\hline $\mathrm{O}(1)$ & $8901(3)$ & $7858(2)$ & $1121(2)$ & $12(1)$ \\
\hline $\mathrm{O}(2)$ & $8597(3)$ & $9266(2)$ & $2712(2)$ & $13(1)$ \\
\hline $\mathrm{O}(3)$ & 4939(3) & $3019(2)$ & $907(2)$ & $14(1)$ \\
\hline $\mathrm{O}(4)$ & $4416(3)$ & $2887(2)$ & $2629(2)$ & $12(1)$ \\
\hline $\mathrm{O}(5)$ & $10652(3)$ & $6967(2)$ & $-2650(2)$ & $12(1)$ \\
\hline $\mathrm{O}(6)$ & $10140(3)$ & $6797(2)$ & $-936(2)$ & $13(1)$ \\
\hline $\mathrm{O}(7)$ & $6048(3)$ & 1993(2) & $-1191(2)$ & $13(1)$ \\
\hline $\mathrm{O}(8)$ & $6403(3)$ & $605(2)$ & $-2776(2)$ & $15(1)$ \\
\hline $\mathrm{O}(9)$ & $2253(4)$ & $379(2)$ & $463(2)$ & $37(1)$ \\
\hline $\mathrm{O}(10)$ & $1550(3)$ & $1210(2)$ & $2982(2)$ & $15(1)$ \\
\hline $\mathrm{O}(11)$ & $6415(3)$ & $882(2)$ & $1946(3)$ & $32(1)$ \\
\hline $\mathrm{O}(12)$ & $8049(3)$ & $4469(2)$ & $-930(2)$ & $10(1)$ \\
\hline $\mathrm{O}(13)$ & $6970(3)$ & $5370(2)$ & $880(2)$ & $11(1)$ \\
\hline $\mathrm{C}(11)$ & $7636(4)$ & $7083(3)$ & $2651(2)$ & $10(1)$ \\
\hline$C(12)$ & $6904(4)$ & $5777(3)$ & $2024(2)$ & $10(1)$ \\
\hline$C(13)$ & $6079(4)$ & $4898(2)$ & $2589(2)$ & $9(1)$ \\
\hline$C(14)$ & $6121(4)$ & $5343(3)$ & $3788(2)$ & $11(1)$ \\
\hline$C(15)$ & $6894(4)$ & $6599(3)$ & $4412(2)$ & $13(1)$ \\
\hline$C(16)$ & $7610(4)$ & $7471(3)$ & $3842(2)$ & $12(1)$ \\
\hline $\mathrm{C}(21)$ & $8860(4)$ & $4960(3)$ & $-2649(2)$ & $10(1)$ \\
\hline $\mathrm{C}(22)$ & $8048(4)$ & $4083(3)$ & $-2084(2)$ & $9(1)$ \\
\hline $\mathrm{C}(23)$ & $7295(4)$ & $2780(3)$ & $-2715(2)$ & $11(1)$ \\
\hline $\mathrm{C}(24)$ & $7297(4)$ & $2400(3)$ & $-3909(2)$ & $12(1)$ \\
\hline$C(25)$ & 7996(4) & $3275(3)$ & $-4483(2)$ & $13(1)$ \\
\hline$C(26)$ & $8790(4)$ & $4537(3)$ & $-3849(2)$ & $12(1)$ \\
\hline$C(110)$ & $8423(4)$ & $8119(3)$ & $2124(2)$ & $11(1)$ \\
\hline$C(130)$ & $5117(4)$ & $3530(3)$ & $2000(2)$ & $11(1)$ \\
\hline$C(210)$ & $9923(4)$ & $6319(3)$ & $-2047(2)$ & $11(1)$ \\
\hline
\end{tabular}


Table S15. Bond lengths $[\AA]$ for $\mathrm{C}_{32} \mathrm{H}_{24} \mathrm{Cu}_{6} \mathrm{O}_{26}$.

\begin{tabular}{llll}
\hline $\mathrm{Cu}(1)-\mathrm{O}(7)$ & $1.8846(19)$ & $\mathrm{O}(8)-\mathrm{C}(230)$ & $1.262(3)$ \\
$\mathrm{Cu}(1)-\mathrm{O}(3)$ & $1.8980(19)$ & $\mathrm{O}(8)-\mathrm{Cu}(3)^{a}$ & $1.9500(19)$ \\
$\mathrm{Cu}(1)-\mathrm{O}(12)$ & $1.9267(18)$ & $\mathrm{O}(13)-\mathrm{C}(12)$ & $1.355(3)$ \\
$\mathrm{Cu}(1)-\mathrm{O}(13)$ & $1.9295(18)$ & $\mathrm{O}(12)-\mathrm{C}(22)$ & $1.354(3)$ \\
$\mathrm{Cu}(1)-\mathrm{Cu}(2)$ & $2.9714(5)$ & $\mathrm{C}(11)-\mathrm{C}(16)$ & $1.401(4)$ \\
$\mathrm{Cu}(2)-\mathrm{O}(1)$ & $1.8840(19)$ & $\mathrm{C}(11)-\mathrm{C}(12)$ & $1.412(4)$ \\
$\mathrm{Cu}(2)-\mathrm{O}(6)$ & $1.8969(19)$ & $\mathrm{C}(11)-\mathrm{C}(110)$ & $1.505(4)$ \\
$\mathrm{Cu}(2)-\mathrm{O}(13)$ & $1.9244(18)$ & $\mathrm{C}(12)-\mathrm{C}(13)$ & $1.416(3)$ \\
$\mathrm{Cu}(2)-\mathrm{O}(12)$ & $1.9334(18)$ & $\mathrm{C}(13)-\mathrm{C}(14)$ & $1.405(4)$ \\
$\mathrm{Cu}(3)-\mathrm{O}(8)^{a}$ & $1.9500(19)$ & $\mathrm{C}(13)-\mathrm{C}(130)$ & $1.490(4)$ \\
$\mathrm{Cu}(3)-\mathrm{O}(10)$ & $1.950(2)$ & $\mathrm{C}(14)-\mathrm{C}(15)$ & $1.371(4)$ \\
$\mathrm{Cu}(3)-\mathrm{O}(11)$ & $1.952(2)$ & $\mathrm{C}(15)-\mathrm{C}(16)$ & $1.386(4)$ \\
$\mathrm{Cu}(3)-\mathrm{O}(4)$ & $1.9647(18)$ & $\mathrm{C}(21)-\mathrm{C}(26)$ & $1.400(4)$ \\
$\mathrm{Cu}(3)-\mathrm{O}(9)$ & $2.216(3)$ & $\mathrm{C}(21)-\mathrm{C}(22)$ & $1.411(4)$ \\
$\mathrm{O}(1)-\mathrm{C}(110)$ & $1.288(3)$ & $\mathrm{C}(21)-\mathrm{C}(210)$ & $1.503(4)$ \\
$\mathrm{O}(2)-\mathrm{C}(110)$ & $1.252(3)$ & $\mathrm{C}(22)-\mathrm{C}(23)$ & $1.413(4)$ \\
$\mathrm{O}(3)-\mathrm{C}(130)$ & $1.280(3)$ & $\mathrm{C}(23)-\mathrm{C}(24)$ & $1.401(4)$ \\
$\mathrm{O}(4)-\mathrm{C}(130)$ & $1.262(3)$ & $\mathrm{C}(23)-\mathrm{C}(230)$ & $1.505(4)$ \\
$\mathrm{O}(5)-\mathrm{C}(210)$ & $1.248(3)$ & $\mathrm{C}(24)-\mathrm{C}(25)$ & $1.388(4)$ \\
$\mathrm{O}(6)-\mathrm{C}(210)$ & $1.291(3)$ & $\mathrm{C}(25)-\mathrm{C}(26)$ & $1.383(4)$ \\
$\mathrm{O}(7)-\mathrm{C}(230)$ & $1.266(3)$ & & \\
\hline $\mathrm{S}$ & & &
\end{tabular}

Symmetry transformations used to generate equivalent atoms:

${ }^{a}-x+1,-y,-z$ 
Table S16. Bond angles [ $\left.{ }^{\circ}\right]$ for $\mathrm{C}_{32} \mathrm{H}_{24} \mathrm{Cu}_{6} \mathrm{O}_{26}$.

\begin{tabular}{|c|c|c|c|}
\hline $\mathrm{O}(7)-\mathrm{Cu}(1)-\mathrm{O}(3)$ & $95.27(8)$ & $\mathrm{C}(130)-\mathrm{O}(3)-\mathrm{Cu}(1)$ & $126.16(17)$ \\
\hline $\mathrm{O}(7)-\mathrm{Cu}(1)-\mathrm{O}(12)$ & $93.37(8)$ & $\mathrm{C}(130)-\mathrm{O}(4)-\mathrm{Cu}(3)$ & $126.70(17)$ \\
\hline $\mathrm{O}(3)-\mathrm{Cu}(1)-\mathrm{O}(12)$ & $171.28(8)$ & $\mathrm{C}(210)-\mathrm{O}(6)-\mathrm{Cu}(2)$ & $126.77(17)$ \\
\hline $\mathrm{O}(7)-\mathrm{Cu}(1)-\mathrm{O}(13)$ & $171.89(8)$ & $\mathrm{C}(230)-\mathrm{O}(7)-\mathrm{Cu}(1)$ & $127.52(17)$ \\
\hline $\mathrm{O}(3)-\mathrm{Cu}(1)-\mathrm{O}(13)$ & $92.07(8)$ & $\mathrm{C}(230)-\mathrm{O}(8)-\mathrm{Cu}(3)^{a}$ & $129.34(18)$ \\
\hline $\mathrm{O}(12)-\mathrm{Cu}(1)-\mathrm{O}(13)$ & $79.23(8)$ & $\mathrm{C}(12)-\mathrm{O}(13)-\mathrm{Cu}(2)$ & $126.82(16)$ \\
\hline $\mathrm{O}(7)-\mathrm{Cu}(1)-\mathrm{Cu}(2)$ & $133.08(6)$ & $\mathrm{C}(12)-\mathrm{O}(13)-\mathrm{Cu}(1)$ & $127.37(16)$ \\
\hline $\mathrm{O}(3)-\mathrm{Cu}(1)-\mathrm{Cu}(2)$ & $131.56(6)$ & $\mathrm{Cu}(2)-\mathrm{O}(13)-\mathrm{Cu}(1)$ & $100.89(8)$ \\
\hline $\mathrm{O}(12)-\mathrm{Cu}(1)-\mathrm{Cu}(2)$ & $39.75(5)$ & $\mathrm{C}(22)-\mathrm{O}(12)-\mathrm{Cu}(1)$ & $127.00(16)$ \\
\hline $\mathrm{O}(13)-\mathrm{Cu}(1)-\mathrm{Cu}(2)$ & $39.49(5)$ & $\mathrm{C}(22)-\mathrm{O}(12)-\mathrm{Cu}(2)$ & $127.04(16)$ \\
\hline $\mathrm{O}(1)-\mathrm{Cu}(2)-\mathrm{O}(6)$ & $95.90(8)$ & $\mathrm{Cu}(1)-\mathrm{O}(12)-\mathrm{Cu}(2)$ & $100.67(8)$ \\
\hline $\mathrm{O}(1)-\mathrm{Cu}(2)-\mathrm{O}(13)$ & $93.45(8)$ & $C(16)-C(11)-C(12)$ & $119.0(2)$ \\
\hline $\mathrm{O}(6)-\mathrm{Cu}(2)-\mathrm{O}(13)$ & $170.65(8)$ & $C(16)-C(11)-C(110)$ & $116.5(2)$ \\
\hline $\mathrm{O}(1)-\mathrm{Cu}(2)-\mathrm{O}(12)$ & $172.63(8)$ & $C(12)-C(11)-C(110)$ & $124.5(2)$ \\
\hline $\mathrm{O}(6)-\mathrm{Cu}(2)-\mathrm{O}(12)$ & $91.46(8)$ & $\mathrm{O}(13)-\mathrm{C}(12)-\mathrm{C}(11)$ & $120.1(2)$ \\
\hline $\mathrm{O}(13)-\mathrm{Cu}(2)-\mathrm{O}(12)$ & $79.19(8)$ & $\mathrm{O}(13)-\mathrm{C}(12)-\mathrm{C}(13)$ & $120.1(2)$ \\
\hline $\mathrm{O}(1)-\mathrm{Cu}(2)-\mathrm{Cu}(1)$ & $133.06(6)$ & $\mathrm{C}(11)-\mathrm{C}(12)-\mathrm{C}(13)$ & $119.8(2)$ \\
\hline $\mathrm{O}(6)-\mathrm{Cu}(2)-\mathrm{Cu}(1)$ & $131.03(6)$ & $\mathrm{C}(14)-\mathrm{C}(13)-\mathrm{C}(12)$ & $118.3(2)$ \\
\hline $\mathrm{O}(13)-\mathrm{Cu}(2)-\mathrm{Cu}(1)$ & $39.62(5)$ & $C(14)-C(13)-C(130)$ & $116.9(2)$ \\
\hline $\mathrm{O}(12)-\mathrm{Cu}(2)-\mathrm{Cu}(1)$ & $39.58(5)$ & $C(12)-C(13)-C(130)$ & $124.8(2)$ \\
\hline $\mathrm{O}(8)^{a}-\mathrm{Cu}(3)-\mathrm{O}(10)$ & $83.46(8)$ & $C(15)-C(14)-C(13)$ & $122.1(2)$ \\
\hline $\mathrm{O}(8)^{a}-\mathrm{Cu}(3)-\mathrm{O}(11)$ & $93.71(9)$ & $\mathrm{C}(14)-\mathrm{C}(15)-\mathrm{C}(16)$ & 119.3(3) \\
\hline $\mathrm{O}(10)-\mathrm{Cu}(3)-\mathrm{O}(11)$ & $168.69(11)$ & $\mathrm{C}(15)-\mathrm{C}(16)-\mathrm{C}(11)$ & $121.3(2)$ \\
\hline $\mathrm{O}(8)^{a}-\mathrm{Cu}(3)-\mathrm{O}(4)$ & $152.98(8)$ & $\mathrm{C}(26)-\mathrm{C}(21)-\mathrm{C}(22)$ & $119.0(2)$ \\
\hline $\mathrm{O}(10)-\mathrm{Cu}(3)-\mathrm{O}(4)$ & $86.66(8)$ & $C(26)-C(21)-C(210)$ & $116.2(2)$ \\
\hline $\mathrm{O}(11)-\mathrm{Cu}(3)-\mathrm{O}(4)$ & $91.08(9)$ & $C(22)-C(21)-C(210)$ & $124.8(2)$ \\
\hline $\mathrm{O}(8)^{a}-\mathrm{Cu}(3)-\mathrm{O}(9)$ & $103.46(9)$ & $\mathrm{O}(12)-\mathrm{C}(22)-\mathrm{C}(21)$ & $120.5(2)$ \\
\hline $\mathrm{O}(10)-\mathrm{Cu}(3)-\mathrm{O}(9)$ & $96.01(10)$ & $\mathrm{O}(12)-\mathrm{C}(22)-\mathrm{C}(23)$ & $119.7(2)$ \\
\hline $\mathrm{O}(11)-\mathrm{Cu}(3)-\mathrm{O}(9)$ & $95.29(12)$ & $\mathrm{C}(21)-\mathrm{C}(22)-\mathrm{C}(23)$ & $119.8(2)$ \\
\hline $\mathrm{O}(4)-\mathrm{Cu}(3)-\mathrm{O}(9)$ & $102.55(9)$ & $\mathrm{C}(24)-\mathrm{C}(23)-\mathrm{C}(22)$ & $118.9(2)$ \\
\hline $\mathrm{C}(110)-\mathrm{O}(1)-\mathrm{Cu}(2)$ & $127.92(17)$ & $\mathrm{C}(24)-\mathrm{C}(23)-\mathrm{C}(230)$ & $117.4(2)$ \\
\hline
\end{tabular}


Table S17. Bond angles [ $\left.{ }^{\circ}\right]$ for $\mathrm{C}_{32} \mathrm{H}_{24} \mathrm{Cu}_{6} \mathrm{O}_{26}$ (continued).

\begin{tabular}{llll}
\hline $\mathrm{C}(22)-\mathrm{C}(23)-\mathrm{C}(230)$ & $123.7(2)$ & $\mathrm{O}(4)-\mathrm{C}(130)-\mathrm{C}(13)$ & $116.6(2)$ \\
$\mathrm{C}(25)-\mathrm{C}(24)-\mathrm{C}(23)$ & $121.5(3)$ & $\mathrm{O}(3)-\mathrm{C}(130)-\mathrm{C}(13)$ & $122.9(2)$ \\
$\mathrm{C}(26)-\mathrm{C}(25)-\mathrm{C}(24)$ & $119.0(3)$ & $\mathrm{O}(5)-\mathrm{C}(210)-\mathrm{O}(6)$ & $120.6(2)$ \\
$\mathrm{C}(25)-\mathrm{C}(26)-\mathrm{C}(21)$ & $121.7(2)$ & $\mathrm{O}(5)-\mathrm{C}(210)-\mathrm{C}(21)$ & $117.8(2)$ \\
$\mathrm{O}(2)-\mathrm{C}(110)-\mathrm{O}(1)$ & $120.9(2)$ & $\mathrm{O}(6)-\mathrm{C}(210)-\mathrm{C}(21)$ & $121.6(2)$ \\
$\mathrm{O}(2)-\mathrm{C}(110)-\mathrm{C}(11)$ & $116.7(2)$ & $\mathrm{O}(8)-\mathrm{C}(230)-\mathrm{O}(7)$ & $121.6(2)$ \\
$\mathrm{O}(1)-\mathrm{C}(110)-\mathrm{C}(11)$ & $122.5(2)$ & $\mathrm{O}(8)-\mathrm{C}(230)-\mathrm{C}(23)$ & $114.9(2)$ \\
$\mathrm{O}(4)-\mathrm{C}(130)-\mathrm{O}(3)$ & $120.5(2)$ & $\mathrm{O}(7)-\mathrm{C}(230)-\mathrm{C}(23)$ & $123.5(2)$ \\
\hline
\end{tabular}

Symmetry transformations used to generate equivalent atoms:

${ }^{a}-x+1,-y,-z$ 
Table S18. Anisotropic displacement parameters $\left[\AA^{2} \times 10^{3}\right]$ for $\mathrm{C}_{32} \mathrm{H}_{24} \mathrm{Cu}_{6} \mathrm{O}_{26}$. The anisotropic displacement factor exponent takes the form: $-2 \pi^{2}\left[\left(h a^{*}\right)^{2} U_{11}+\ldots+2 h k a^{*} b^{*} U_{12}\right]$.

\begin{tabular}{|c|c|c|c|c|c|c|}
\hline & $U_{11}$ & $U_{22}$ & $U_{33}$ & $U_{23}$ & $U_{13}$ & $U_{12}$ \\
\hline $\mathrm{Cu}(1)$ & $14(1)$ & $7(1)$ & $11(1)$ & $3(1)$ & $4(1)$ & $-1(1)$ \\
\hline $\mathrm{Cu}(2)$ & $13(1)$ & $7(1)$ & $12(1)$ & $4(1)$ & $4(1)$ & $-1(1)$ \\
\hline $\mathrm{Cu}(3)$ & $15(1)$ & $6(1)$ & $20(1)$ & $5(1)$ & $7(1)$ & 1(1) \\
\hline $\mathrm{O}(1)$ & $17(1)$ & $9(1)$ & $14(1)$ & $5(1)$ & $6(1)$ & $2(1)$ \\
\hline $\mathrm{O}(2)$ & $16(1)$ & $7(1)$ & $17(1)$ & $3(1)$ & $6(1)$ & $0(1)$ \\
\hline $\mathrm{O}(3)$ & $17(1)$ & $10(1)$ & $13(1)$ & $3(1)$ & $4(1)$ & $-2(1)$ \\
\hline $\mathrm{O}(4)$ & $16(1)$ & $6(1)$ & $15(1)$ & $4(1)$ & $6(1)$ & $0(1)$ \\
\hline $\mathrm{O}(5)$ & $15(1)$ & $9(1)$ & $15(1)$ & $6(1)$ & $6(1)$ & $0(1)$ \\
\hline $\mathrm{O}(6)$ & $16(1)$ & $9(1)$ & $14(1)$ & $4(1)$ & $4(1)$ & $-2(1)$ \\
\hline $\mathrm{O}(7)$ & $19(1)$ & $8(1)$ & $12(1)$ & $5(1)$ & $5(1)$ & $0(1)$ \\
\hline $\mathrm{O}(8)$ & $20(1)$ & $5(1)$ & $19(1)$ & $3(1)$ & $6(1)$ & $0(1)$ \\
\hline $\mathrm{O}(9)$ & $66(2)$ & $20(1)$ & $21(1)$ & $7(1)$ & $3(1)$ & $-4(1)$ \\
\hline $\mathrm{O}(10)$ & $15(1)$ & $6(1)$ & $28(1)$ & $7(1)$ & $8(1)$ & $2(1)$ \\
\hline $\mathrm{O}(11)$ & $22(1)$ & $14(1)$ & $68(2)$ & $19(1)$ & $19(1)$ & $6(1)$ \\
\hline $\mathrm{O}(12)$ & $15(1)$ & $6(1)$ & $10(1)$ & $4(1)$ & $4(1)$ & $-2(1)$ \\
\hline $\mathrm{O}(13)$ & $14(1)$ & $8(1)$ & $12(1)$ & $4(1)$ & $5(1)$ & $0(1)$ \\
\hline$C(11)$ & $9(1)$ & $9(1)$ & $13(1)$ & $5(1)$ & $2(1)$ & $2(1)$ \\
\hline$C(12)$ & $9(1)$ & $10(1)$ & $12(1)$ & $6(1)$ & $3(1)$ & $4(1)$ \\
\hline$C(13)$ & $7(1)$ & $8(1)$ & $13(1)$ & $5(1)$ & $3(1)$ & $2(1)$ \\
\hline$C(14)$ & $11(1)$ & $10(1)$ & $14(1)$ & $6(1)$ & $5(1)$ & $3(1)$ \\
\hline$C(15)$ & $16(1)$ & $13(1)$ & $11(1)$ & $4(1)$ & $4(1)$ & $3(1)$ \\
\hline$C(16)$ & $14(1)$ & $8(1)$ & $14(1)$ & $1(1)$ & $4(1)$ & $2(1)$ \\
\hline$C(21)$ & $10(1)$ & $9(1)$ & $14(1)$ & $6(1)$ & $3(1)$ & $4(1)$ \\
\hline$C(22)$ & $8(1)$ & $10(1)$ & $11(1)$ & $4(1)$ & $2(1)$ & $4(1)$ \\
\hline$C(23)$ & $11(1)$ & $10(1)$ & $13(1)$ & $4(1)$ & $2(1)$ & $2(1)$ \\
\hline$C(24)$ & $12(1)$ & $10(1)$ & $13(1)$ & $4(1)$ & $1(1)$ & $2(1)$ \\
\hline$C(25)$ & $14(1)$ & $15(1)$ & $13(1)$ & $5(1)$ & $3(1)$ & $4(1)$ \\
\hline$C(26)$ & $11(1)$ & $13(1)$ & $14(1)$ & $8(1)$ & $3(1)$ & $4(1)$ \\
\hline$C(110)$ & $9(1)$ & $9(1)$ & $17(1)$ & $5(1)$ & $2(1)$ & $2(1)$ \\
\hline$C(130)$ & $11(1)$ & $8(1)$ & $15(1)$ & $4(1)$ & $5(1)$ & $3(1)$ \\
\hline
\end{tabular}




\begin{tabular}{rrrrrrr}
$\mathrm{Cu}(210)$ & $9(1)$ & $11(1)$ & $16(1)$ & $7(1)$ & $4(1)$ & $5(1)$ \\
$\mathrm{Cu}(230)$ & $9(1)$ & $9(1)$ & $15(1)$ & $4(1)$ & $-1(1)$ & $2(1)$ \\
\hline
\end{tabular}

Table S19. Hydrogen coordinates $\left[\times 10^{4}\right]$ and isotropic displacement parameters $\left[\AA^{2} \times 10^{3}\right]$ for $\mathrm{C}_{32} \mathrm{H}_{24} \mathrm{Cu}_{6} \mathrm{O}_{26}$.

\begin{tabular}{lcccc}
\hline & $x$ & $y$ & $z$ & $U(e q)$ \\
\hline $\mathrm{H}(9 \mathrm{~A})$ & $1100(30)$ & $200(40)$ & $470(40)$ & 56 \\
$\mathrm{H}(9 \mathrm{~B})$ & $2350(60)$ & $880(30)$ & $60(30)$ & 56 \\
$\mathrm{H}(10 \mathrm{~A})$ & $970(40)$ & $1750(20)$ & $2780(30)$ & 23 \\
$\mathrm{H}(10 \mathrm{~B})$ & $660(40)$ & $570(20)$ & $2860(30)$ & 23 \\
$\mathrm{H}(11 \mathrm{~A})$ & $7210(50)$ & $1560(20)$ & $2120(40)$ & 47 \\
$\mathrm{H}(11 \mathrm{~B})$ & $7070(50)$ & $340(30)$ & $2100(40)$ & 47 \\
$\mathrm{H}(14)$ & 5596 & 4756 & 4178 & 13 \\
$\mathrm{H}(15)$ & 6939 & 6868 & 5226 & 15 \\
$\mathrm{H}(16)$ & 8092 & 8349 & 4266 & 14 \\
$\mathrm{H}(24)$ & 6809 & 1523 & -4336 & 14 \\
$\mathrm{H}(25)$ & 7930 & 3012 & -5301 & 16 \\
$\mathrm{H}(26)$ & 9300 & 5132 & -4236 & 14 \\
\hline
\end{tabular}


Table S20. Hydrogen bond lengths $[\AA]$ and angles $\left[{ }^{\circ}\right]$ for $\mathrm{C}_{32} \mathrm{H}_{24} \mathrm{Cu}_{6} \mathrm{O}_{26}$.

\begin{tabular}{lcccc}
\hline $\mathrm{D}-\mathrm{H} \cdots \mathrm{A}$ & $d(\mathrm{D}-\mathrm{H})$ & $d(\mathrm{H} \cdots \mathrm{A})$ & $d(\mathrm{D} \cdots \mathrm{A})$ & $<(\mathrm{DHA})$ \\
\hline $\mathrm{O}(9)-\mathrm{H}(9 \mathrm{~A}) \cdots \mathrm{O}(9)^{b}$ & $0.809(18)$ & $2.38(3)$ & $3.119(6)$ & $153(4)$ \\
$\mathrm{O}(9)-\mathrm{H}(9 \mathrm{~B}) \cdots \mathrm{O}(1)^{c}$ & $0.820(18)$ & $2.36(3)$ & $3.115(3)$ & $154(4)$ \\
$\mathrm{O}(9)-\mathrm{H}(9 \mathrm{~B}) \cdots \mathrm{O}(3)$ & $0.820(18)$ & $2.61(4)$ & $3.080(3)$ & $118(3)$ \\
$\mathrm{O}(10)-\mathrm{H}(10 \mathrm{~A}) \cdots \mathrm{O}(5)^{c}$ & $0.821(17)$ & $1.946(19)$ & $2.752(3)$ & $166(3)$ \\
$\mathrm{O}(10)-\mathrm{H}(10 \mathrm{~B}) \cdots \mathrm{O}(2)^{d}$ & $0.837(17)$ & $1.833(18)$ & $2.667(3)$ & $174(3)$ \\
$\mathrm{O}(11)-\mathrm{H}(11 \mathrm{~A}) \cdots \mathrm{O}(5)^{e}$ & $0.823(18)$ & $1.93(2)$ & $2.751(3)$ & $171(4)$ \\
$\mathrm{O}(11)-\mathrm{H}(11 \mathrm{~B}) \cdots \mathrm{O}(2)^{f}$ & $0.836(18)$ & $1.89(2)$ & $2.723(3)$ & $171(4)$ \\
\hline
\end{tabular}

Symmetry transformations used to generate equivalent atoms:
${ }^{a}-x+1,-y,-z$
${ }^{e}-x+2,-y+1,-z$
${ }^{b}-x,-y,-z$
${ }^{c}-x+1,-y+1,-z$
${ }^{d} X-1, y-1, z$
$f_{x, y-1, z}$ 